\title{
STRUCTURE OF MARTENSITIC CARBON STEELS AND CHANGES IN THE MICROSTRUCTURE WHICH OCCUR UPON TEMPERING.
}

\author{
By H. S. Rawdon and S. Epstein.
}

ABSTRACT.

A study of the changes in structure resulting upon tempering was made in a series of 6 carbon steels ranging from 0.07 to 1.12 per cent carbon, quenched from temperatures varying from 750 to $1250^{\circ} \mathrm{C}$. and tempered for different lengths of time at roo to $650^{\circ} \mathrm{C}$. Upon quenching, martensite is formed throughout each austenite crystal in a manner strictly analogous to the freezing of solid-solution alloys. A redistribution of carbon takes place and the conspicuous martensite plates are found to be distinctly lower in carbon than the "filling material" between the plates. The enrichment of the carbon in the "filling material" may be great enough in some steels as to allow small patches of austenite to persist after quenching.

The changes in structure and in hardness which occur during tempering vary in character according to whether they take place above or below $250^{\circ} \mathrm{C}$. Below this temperature, the structural changes are relatively slight and are best revealed in the higher carbon steels by an alkaline oxidizing etching reagent or by immersion of a polished specimen in liquid air. At approximately $250^{\circ}$ martensite and austenite, if present, undergo a transformation and the steel assumes a granular structural appearance and reacts vigorously toward dilute acid etching reagents. The granules, cementite particles, grow in size progressively as the tempering temperature above $250^{\circ}$ is increased.

Hardened steels, tempered by heating to temperatures below $250^{\circ}$, show a slight decrease in hardness; above this temperature, the decrease is rapid. Curves are given to illustrate the effect of the tempering period and temperature. Steels containing austenite harden appreciably below $250^{\circ} \mathrm{C}$. with the long tempering periods, though no evidence was obtained to show that the steel regained or exceeded its initial hardness upon tempering as has been reported by other investigators.

\section{CONTENTS}

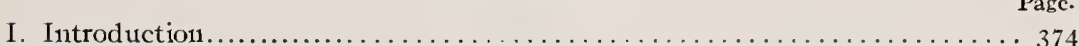

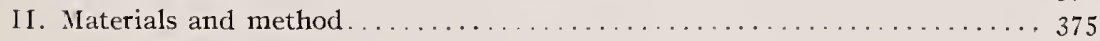

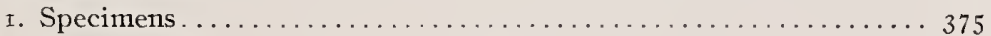

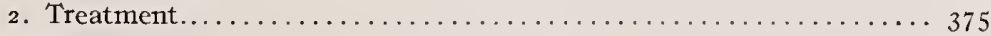

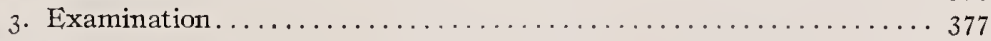

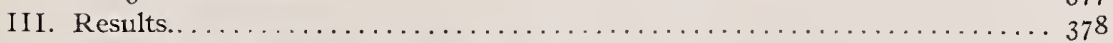

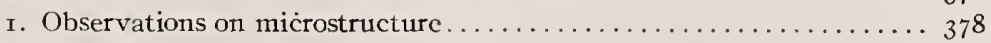

2. Determination of hardness.............................. $3^{81}$ 
IV. Discussion.

I. Microstructure

(a) Structure of martensite........................ 38 I

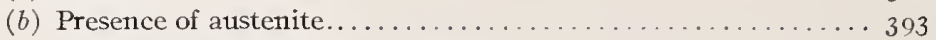

(c) Changes in structure upon heating................. 393

Low-temperature changes................... . 395

Tempering at higher temperatures............. $40 \mathrm{I}$

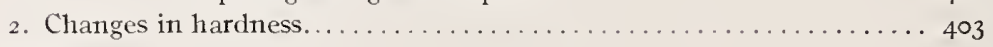

V. Summary.

\section{INTRODUCTION.}

Although the nature of the atomic change occurring in iron during the allotropic transformation-for example, in the change from the gamma modification to the alpha form-is now quite clearly understood, ${ }^{1}$ there still remains much to be learned concerning the visible structural changes, particularly those accompanying the reheating or tempering of a hardened steel. It is by the evidence obtained from the microscopic examination that the physical metallurgist is guided, in large measure. The work herein discussed and summarized was planned primarily as a supplement to the study of the thermal and physical changes accompanying the tempering of hardened steels, the results of which have already been published. ${ }^{2}$ In spite of the great amount of investigational work which has been done upon the hardening and tempering of steel, representative micrographs, showing the structural changes through which the materials pass as the "drawing" operation proceeds, are rather rare in the technical literature. The best work along this line is undoubtedly that of Hanemann. ${ }^{3}$ However, on account of the uncertainty introduced into the results by the lack of care in the choice of the quenching temperature used, further work appeared to be necessary to substantiate some of the conclusions reached by Hanemann. This point will be discussed later at greater length. Incidentally, the present investigation also gave some information bearing on the theory of the cause of quenching cracks in steel recently put forward by Honda, Matsushite, and Idei. ${ }^{4}$

\footnotetext{
${ }^{1}$ A. Westgren, Roentgen Spectrographic Investigation of Iron and Steel; Journ. Iron and Steel Inst., 103, p. 303. Z. Jeffries and R. S. Archer, Slip Interference Theory of the Hardening of Xretals; Chem. and Met. Eng., 24, p. 1057.

${ }^{2}$ H. Scott and H. G. Movius, Thermal and Physical Changes Accompanying the Heating of Hardened Carbon Steels; B. S. Sci. Papers. No. 396; I92I. The results obtained by previous investigators have been carefully summarized by the authors.

${ }^{3}$ H. Hanemann, \letallographie und Wärmebehandlung, I9r5; also Stahl und Eisen, 32, p. 1397; 1912.

${ }^{4} \mathrm{~K}$. Honda and S. Idei, On the Distribution of Hardness in Quenched Carbon SteeIs and Quenching Cracks, Sci. Reports, Tohoku Imp. Univ., Sendai. Japan, 9, p. 49r. Also Honda, Mratsuslite. and Idei On the Cause of Quenching Cracks, Jour. Iron and Steel Inst., 103, p. 251 ; I921.
} 


\section{MATERIALS AND METHOD.}

1. SPECIMENS.

As materials for study, six steels were used the compositions of which are summarized in Table I. Five of these were the same as those used by one of the authors in the investigation of the effect of grain size upon the hardness of annealed steel. ${ }^{5}$ Micrographs showing the structural condition of the various steels, after different annealing conditions, have been given in this article and hence none have been included in the present one.

The investigation was confined entirely to carbon steels, since the introduction of alloying elements renders the microstructure much more complex than it would be otherwise. The structural changes which occur in the special steels are most conveniently studied and described by reference to the conditions which obtain in the simpler carbon steels. The material was in the form of rectangular bars of a cross section of $\mathrm{I}$ by $\mathrm{I} / 2$ inch. Portions $\mathrm{I} / 2$ inches in length were sawed from each bar. These short lengths were then cut by a series of 5 transverse cuts which did not extend entirely through the bar, so that a comblike specimen consisting of six teeth, approximately $\mathrm{r} / 4$ inch thick, resulted. The teeth were spread apart from each other and the "comb" bent into a curve shape so as to permit uniform hardening of all the "teeth." After hardening, the separate teeth of each comb could easily be snapped apart from each other when the individual specimens were needed for further use. Each "comb" furnished 6 small specimens, hardened under identical conditions, for use in tempering at the series of temperatures chosen.

TABLE 1.-Results of Chemical Analysis of Steels Used.

\begin{tabular}{|c|c|c|c|c|c|}
\hline Specimen. & Carbon. & Manganese. & Phosphorus. & Sulphur. & Silicon. \\
\hline $\begin{array}{l}1 \\
2 \\
3 \\
4 \\
4 \\
5 \\
6\end{array}$ & $\begin{array}{r}\text { Per cent. } \\
0.07 \\
.19 \\
.46 \\
.70 \\
.85 \\
1.12\end{array}$ & $\begin{array}{r}\text { Per cent. } \\
0.27 \\
.41 \\
.36 \\
.22 \\
.28 \\
.23\end{array}$ & $\begin{array}{r}\text { Per cent. } \\
0.006 \\
.004 \\
.019 \\
.023 \\
.016 \\
.019\end{array}$ & $\begin{array}{r}\text { Per cent. } \\
0.054 \\
.050 \\
.047 \\
.011 \\
.013 \\
.013\end{array}$ & $\begin{array}{r}\text { Per cent. } \\
0.002 \\
.002 \\
.060 \\
.227 \\
.213 \\
.223\end{array}$ \\
\hline
\end{tabular}

\section{TREATMENT.}

Specimens of the shape described above, representative of each of the steels used, were hardened by quenching in water from various temperatures in the range from $750\left(1380^{\circ} \mathrm{F}\right.$.) to $\mathrm{I} 25^{\circ} \mathrm{C}$.

\footnotetext{
Henry S. Rawdon and Emilio Jimeno-Gil, Relation Between the Brinell Hardness and the Grain Size of Annealed Carbon Steels; B. S. Sci. Papers, No. 397; r920.
} 
$\left(2280^{\circ} \mathrm{F}\right.$.), as shown in Figure I. In no case was it desired to use quenching temperatures higher than the solidus temperatures for austenite of the various compositions used, and $1250^{\circ} \mathrm{C}$. was considered to be as close as it was safe to approach this temperature for the steels of the higher carbon contents. The effect of the period during which each specimen was held at the temperature before quenching was also considered. In the earlier part of the investigation-that is, for the quenching temperatures of $75^{\circ}, 85^{\circ}$,

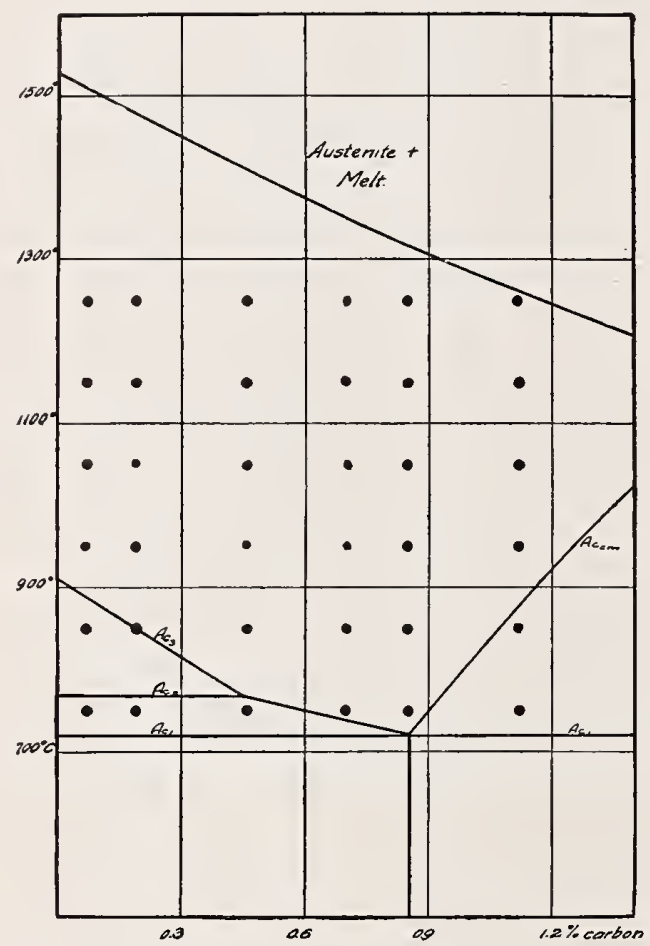

FIG. I.-Portion of the constitutional diagram of the iron-carbon alloys showing the temperatures from which the steels were quenched. and $950^{\circ} \mathrm{C}$. - five periods, $\mathrm{I} / 4, \mathrm{~T} / 2, \mathrm{I}, 2$, and 4 hours, were used. However, for the higher temperatures, IO5O, II 5 O, and $1250^{\circ} \mathrm{C}$., the number was reduced to three, $\mathrm{I} / 2, \mathrm{I}$, and 2 hours.

The specimens were packed in shallow sheetiron boxes, which were then filled with fine silica containing approximately 5 per cent powdered charcoal, to prevent decarburization of the steel during the heating. By means of a wire previously attached, each comb could be withdrawn from the furnace after the desired heating period and quenched in water.

For heating the specimens at the three lower temperatures, 750,850 , and $950^{\circ} \mathrm{C}$., an electric resistance muffle furnace was used, for the remaining temperatures, a gas-fired muffle furnace. The temperatures were measured in each case by means of chromel-alumel thermocouple of I4-gage wire and a portable potentiometer. No difficulty was experienced in regulating the temperature within $5^{\circ}$ of the desired value. A large volume of water was used for quenching the specimens, approximately I 2 to $\mathrm{I}_{5}$ gallons, and the temperature frequently measured after each quenching. This temperature varied between 19 and $22^{\circ} \mathrm{C}$. 
The tempering of the hardened specimens was carried out at the following temperatures: I00, 200, 245,300,400, 500, and $650^{\circ} \mathrm{C}$. $\left(2 \mathrm{I} 2,392,473,572,75^{2}, 932\right.$, and $\mathrm{I} 202^{\circ} \mathrm{F}$.). Previous investigations ${ }^{6}$ have shown the importance of the changes in the neighborhood of $250^{\circ} \mathrm{C}$, , hence the reason for the choice of a tempering temperature approximately midway between 200 and $300^{\circ} \mathrm{C}$. in the series above.

For tempering at $100^{\circ} \mathrm{C}$, the specimens were suspended in the upper part of Erlenmeyer flasks of 3 liters capacity, containing some distilled water, and fitted with reflux condensers through which cold water was passed. The flasks were heated on an electric hot plate for a period of 20 days, the specimens being taken out at intervals for observation and hardness measurements. Several tempering periods were used for each of the other temperatures. These varied from 30 minutes to $4 \frac{1}{2}$ hours for the 200 , 245 , and $300^{\circ} \mathrm{C}$. treatment and from 15 minutes to $2 / 4$ hours for the higher temperatures. For the lower tempering temperatures $\left(200,245\right.$, and $300^{\circ} \mathrm{C}$.) the specimens were suspended in a gasheated oil bath; those at 400 and $500^{\circ} \mathrm{C}$., in a salt bath (sodium and potassium nitrate), and an electric resistance muffle furnace was employed for those at $650^{\circ} \mathrm{C}$.

In order to avoid any possible uncertainty introduced by the repeated tempering of the specimens, new-that is, untempered-samples were used for each of the four lower temperatures $\left(100,200,245\right.$, and $300^{\circ} \mathrm{C}$.). The $100^{\circ}$ specimens were later tempered at $400^{\circ} \mathrm{C}$., the $200^{\circ}$ specimens at $500^{\circ} \mathrm{C}$., and for $650^{\circ} \mathrm{C}$. the specimens previously tempered at $2+5^{\circ} \mathrm{C}$. were used.

\section{EXAMINATION.}

In all cases the specimens were deeply ground on a transverse section before any examination was made, so that the results obtained would be indicative of the condition obtaining throughout the interior of the sample and not merely of the surface.

The size of the specimens, which had purposely been made small, in order to insure vigorous and uniform hardening throughout, permitted only scleroscope hardness determinations, in addition to the study of the microstructure, being made. The hardness of each specimen was determined in its hardened, but untempered, state as a check upon the efficiency and uniformity of the hardening treatment. 
In the preparation of the samples for the study of the microstructure, it was necessary that the etching period be very carefully controlled in order that comparable results be obtained. Two common types of etching reagents were used for revealing the structure, 5 per cent alcoholic solution of picric acid and alkaline sodium picrate solution. On account of the variation in the composition of the steels used, as well as the changes brought about by tempering, which changes affect the etching rate very materially, it was impracticable to attempt to use a single etching period throughout. For the steels of lower carbon content (o.o7, o.I9, and 0.46 per cent), in the quenched and in the slightly tempered states $\left(300^{\circ} \mathrm{C}\right.$. and below), a $3^{\circ}$-second period was used for the picric acid etching. The same steels tempered above $300^{\circ} \mathrm{C}$. were etched for a period of Io seconds. For the remaining steels $(0.70,0.85$, and I.I 2 per cent carbon), the etching period was shortened, I 5 seconds being used for the specimens tempered at the lower temperatures $\left(300^{\circ} \mathrm{C}\right.$. or below), and 5 seconds for those at the higher temperatures. For etching with sodium picrate solution, a period of approximately 20 minutes was used.

Some consideration was also given to the effect of immersion in liquid air as a means of demonstrating the presence of austenite in the hardened high-carbon steels and persistence of this constituent upon tempering. This is discussed later in detail.

\section{RESULTS.}

\section{OBSERVATIONS ON MICROSTRUCTURE.}

On account of the large number of specimens used in the investigation and the still greater number of microscopic observations necessary, it was manifestly impossible to reproduce micrographs showing all the structural conditions found. However, in Figures 2 to $\mathrm{I}_{5}$, inclusive, representative micrographs have been given to illustrate the structures obtained by the hardening treatment and the changes which ensue as the specimens are tempered. As a general rule, the structure is much more striking and pronounced in the steels of high carbon than in those of lower carbon content. For this reason, micrographs of the steel of 0.85 and of I.I 2 per cent carbon content have been given in Figures 4 and 5 to illustrate the structural effect produced by holding the steel for varying periods of time at the quenching temperature before immersing it in the quenching liquid. The general effect of varying the quenching temperature has been shown for the other steels in Figures 2 and 3 . 


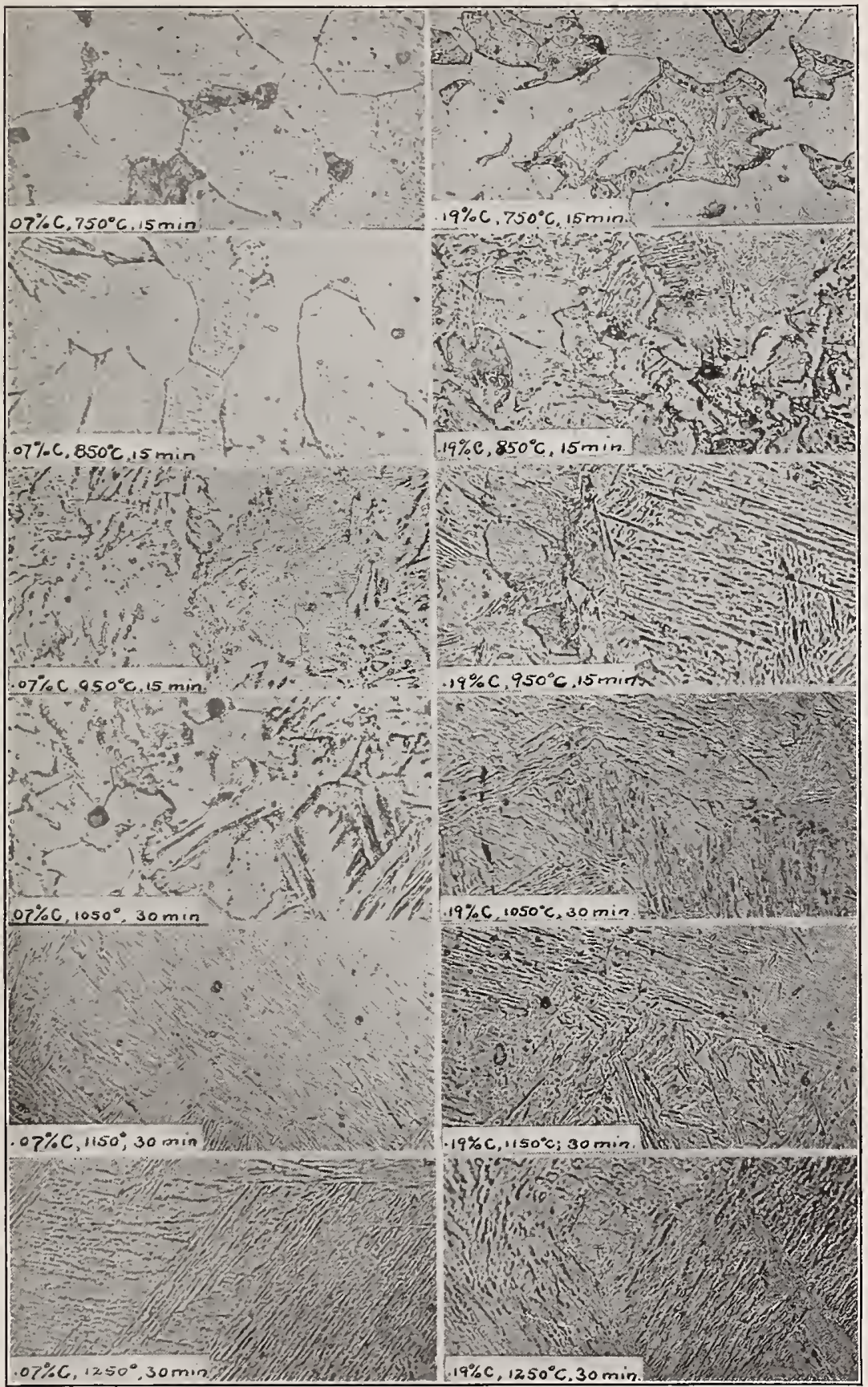

FIG. 2.-Effect of quenching temperature upon the microstructure of 0.07 and 0.10 per cent carbon steel when hardened in water; $\times 500$.

Etching reagent, see p. 378 .

$111668^{\circ}-22-2$ 


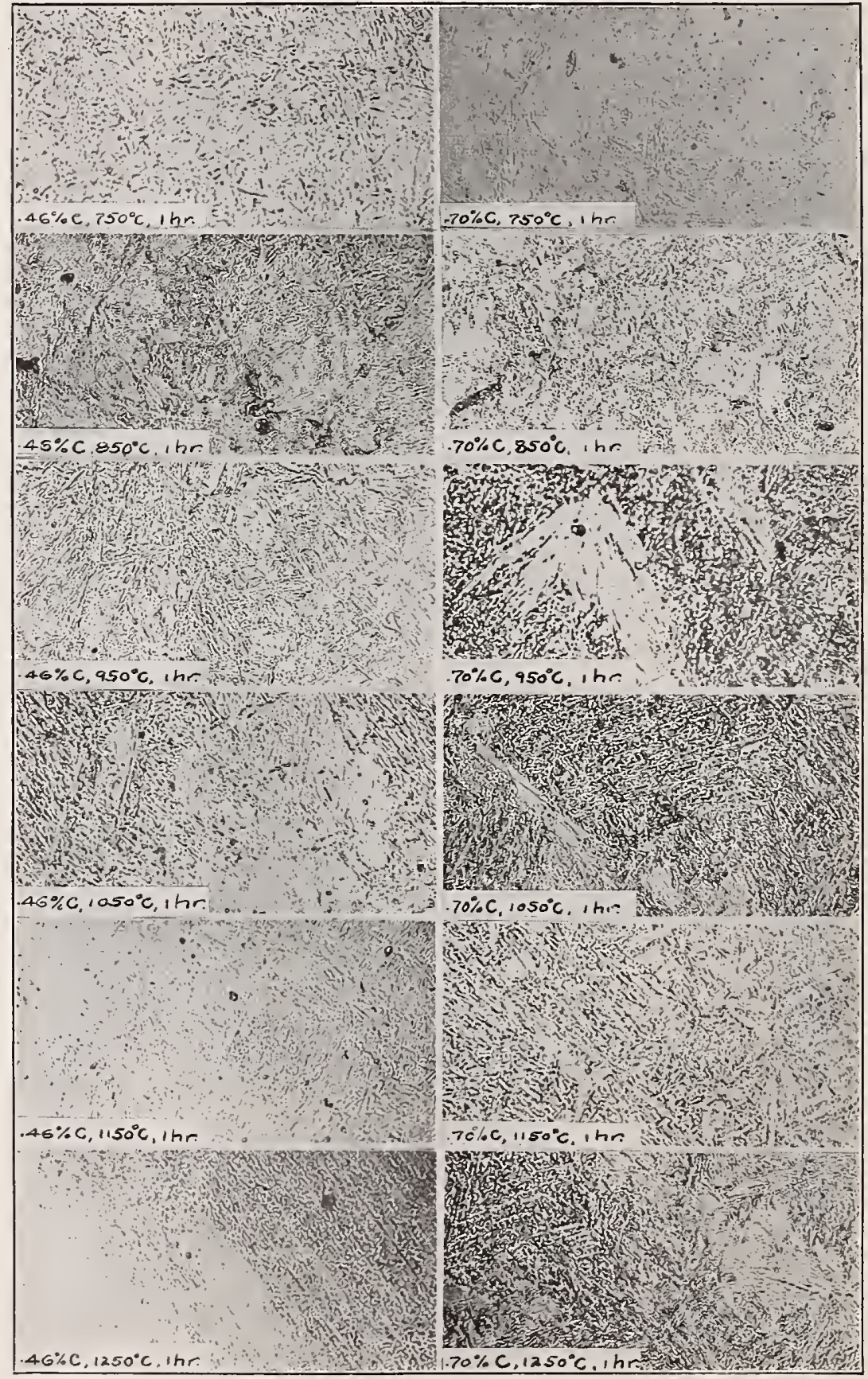

FIG. 3. - Effect of quenching temperature upon the microstructure of 0.76 and 0.70 per cent carbon steel when hardened in water; $\times 500$. 
The effects of tempering upon the structure of the hardened steels have been illustrated in Figures 6 to I $_{5}$, inclusive. In the examination of these micrographs some allowance should be made for the fact that different specimens were used for most of the observations of the microstructure; that is, the same specimen was not submitted in turn to each of the treatments making up a series. Such slight structural features as appear to be peculiar to the individual specimen, rather than characteristic of the general treatment to which the material was subjected, should be disregarded.

\section{DETERMINATION OF HARDNESS.}

A determination of hardness was made upon each of the specimens after quenching. The small size of the individual samples rendered the use of the Brinell method impossible, hence the scleroscope ("recording" instrument) was used throughout. The prime object of the hardness measurements of the quenched specimens was to check the efficiency of the hardening treatment. Specimens which were found to be considerably below the maximum hardness attainable were discarded. The hardness survey also served as a means for detecting the carburization of the two steels of lowest carbon content which occurred upon heating at the highest temperatures for the long periods used. These specimens were discarded also.

The determination of the changes in hardness produced by tempering was of considerable importance in that it confirmed and explained some of the features observed in the microstructure. Figures 17 and 18 have been included as typical of the scleroscope hardiness results obtained and will be discussed later at greater length.

An attempt was made, though without much success, to differentiate between the hardness of the different "constituents" which make up the martensitic structure by means of the "sclerometer."'7

\section{DISCUSSION.}

\section{MICROSTRUCTURE.}

(a) Structure of Martensite.-Although, as previously stated, recent investigations upon the atomic structure of metals have settled most of the contentions concerning the fundamental

i The instrument described by C. H. Bierbatm, A. S. M. F., annual meeting I920; Report of Subcommittee on Bearing Metals, and termed a "microcharacter," was used for this purpose, 
nature of the transformations in iron, there still remains much to be learned concerning the martensitic condition. The results of typical examinations summarized in preceding figures illustrate the effect of progressively increasing the temperature from which the material was quenched and show that, for specimens of the size used in this investigation, the temperature of quenching is the principal factor which determines the coarseness of the needlelike structure. This fact suggests immediately that it is the size of the grains of austenite within which the martensite is formed that determines this structural feature, assuming, of course, that in every case the rate of cooling is such as to favor the formation of martensite within the material. Martensite produced from austenite quenched just after the completion of the transformation, $\mathrm{Ac}_{3}$, is extremely fine and almost structureless in microscopic appearance, since the grain size of the austenite from which it was produced was correspondingly fine. This is most noticeable in steels whose carbon content does not differ very much from that of the eutectoid composition. As the temperature, to which the material is heated before quenching, is raised, the grain size of the austenite increases, giving rise progressively to coarser and longer "needles" of martensite upon quenching. In no case was any evidence obtained which would lead one to believe that a "needle" of martensite may result from the combined action of two or more adjacent grains of austenite. Each austenitic grain gives rise to its own set of "needles" which is quite distinct from that originating from each of the other austenitic grains.

The influence of the length of time for which the specimen was held at the quenching temperature was almost negligible for most cases observed. The shortest period used, i 5 minutes, appeared to be sufficient for the small specimens to attain maximum grain size, so that an increase up to 2 or 4 hours produced only a slight effect upon the resulting structure of the martensite upon quenching. The effect was somewhat more marked in specimens heated at temperatures slightly above the austenitic transformation, the longer period being equivalent in effect to a somewhat higher temperature in that a slightly coarser austenitic grain, and consequently a coarser martensite, resulted. A more noticeable effect was observed in those specimens containing either free ferrite or free cementite at the temperatures mentioned above; a long period was often necessary to permit maximum absorption of the excess constituent into the austenitic solution. (Figs. 2 and 5.) 


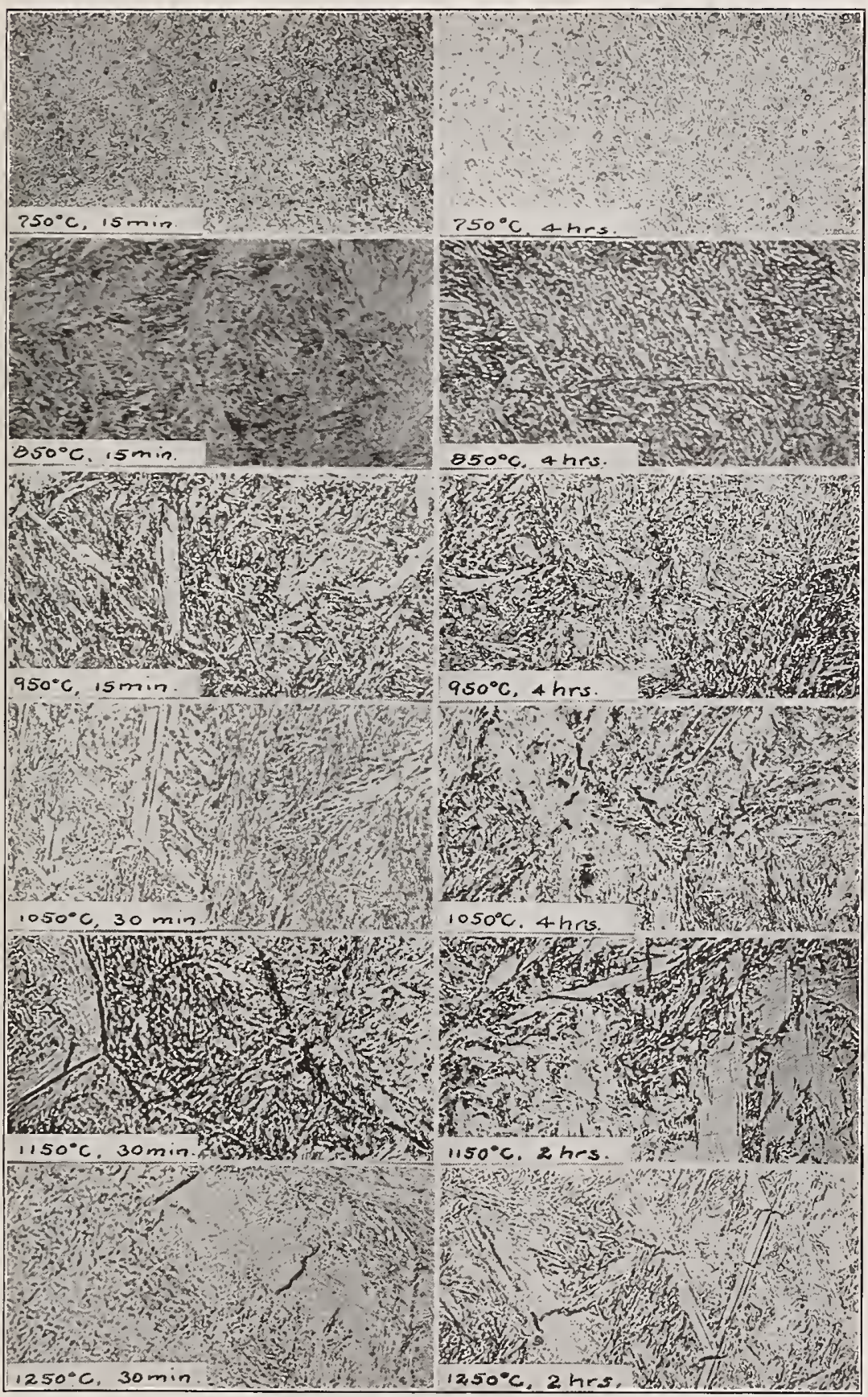

FIG. 4.-Effect of heating period and quenching temperature upon the microstructure of 0.85 per cent carbon stcel when hardened in water; $\times 500$.

Etching reagent, see p. 378 . 


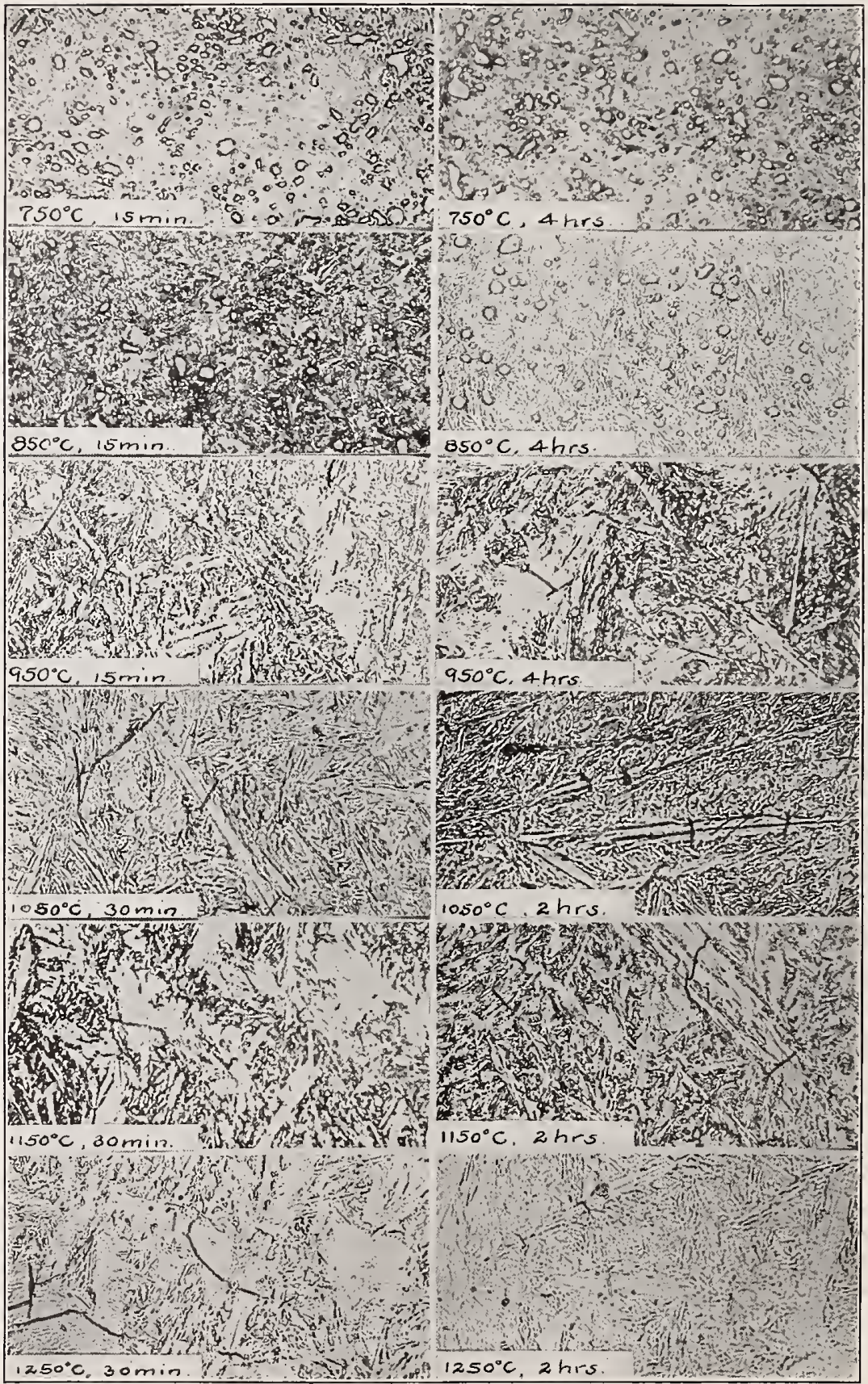

FIG. 5.-Effect of heating period and quenching temperature upon the microstructure of $I . I 2$ per cent carbon steel when hardened in water; $\times 500$. 
Tliis was found to be particularly true for spheroidized cementite, a period of + hours at $85^{\circ} \mathrm{C}$. being insufficient to permit complete absorption of this constituent.

In addition to the coarser "needle size" of the martensite in specimens quenched from the higher temperatures, an additional structural feature may exist in the steels of high carbon content. This is the retention of small patches of austenite. The details of the structural condition are best observed in steels of high carbon content which have been quenched from a very high temperature. By increasing the size of the austenite grains previous to the quenching, the details are exaggerated and thus more readily observed.

There is a very marked similarity between the structure resulting from the transformation of a grain of austenite into martensite and that originating during the freezing of a molten alloy into a solid solution. The selective freezing in the latter case, by which a heterogeneity of structure and composition results throughout each crystal of the solidified alloy, which manifests itself by the presence of branching "cores" of a very different composition from the remainder of the crystal, is well known and needs no further description here. The transformation of a crystal of austenite of uniform composition throughout into a heterogeneous mixture of martensite proceeds in a manner strictly analogous to selective freezing. During this change, along certain planes within each austenite crystal, presumably the cleavage planes, lenticular "plates" of material considerably lower in carbon content than that of the average throughout the crystal are formed. These plates, usually termed "needles," as they have this appearance when sectioned, give to martensite its characteristic appearance. The material filling the space between the needles correspondingly becomes enriched in carbon, and in steels of high carbon content there of ten exist small patches with a carbon content high enough to permit retention of small portions as austenite in the cooled specimen. Between the primary needles of martensite are found smaller needles and at times a darkly etching constituent resembling troostite. (Figs. 4 and 5.) The fact that austenite has been retained in the quenched specimen may often not be apparent in a microscopic examination of the hardened material after etching. However, by tempering the steel at 100 or $200^{\circ} \mathrm{C}$. the patches of austentite, if present, are readliy seen upon etching. (Fig. r 2.) Immersion of the specimens in liquid air may also reveal the pres- 


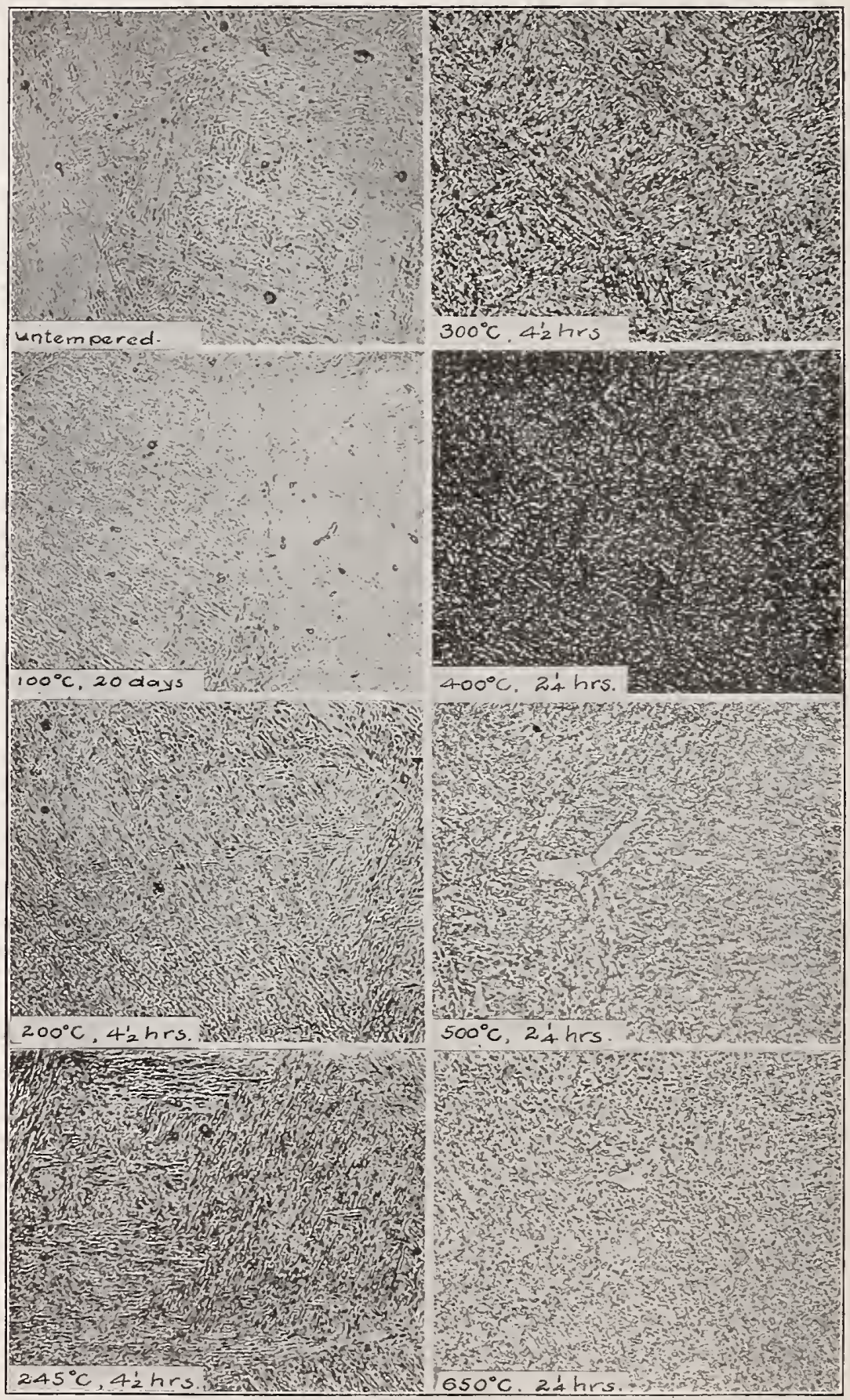

FIG. 6. - Effect of tempering upon the microstructure of o. 60 per cent carbon steel quenched in water from $1050^{\circ} \mathrm{C}$. after 2 hours at this temperature; $\times 500$.

Etching reagent, see p. 378 . 


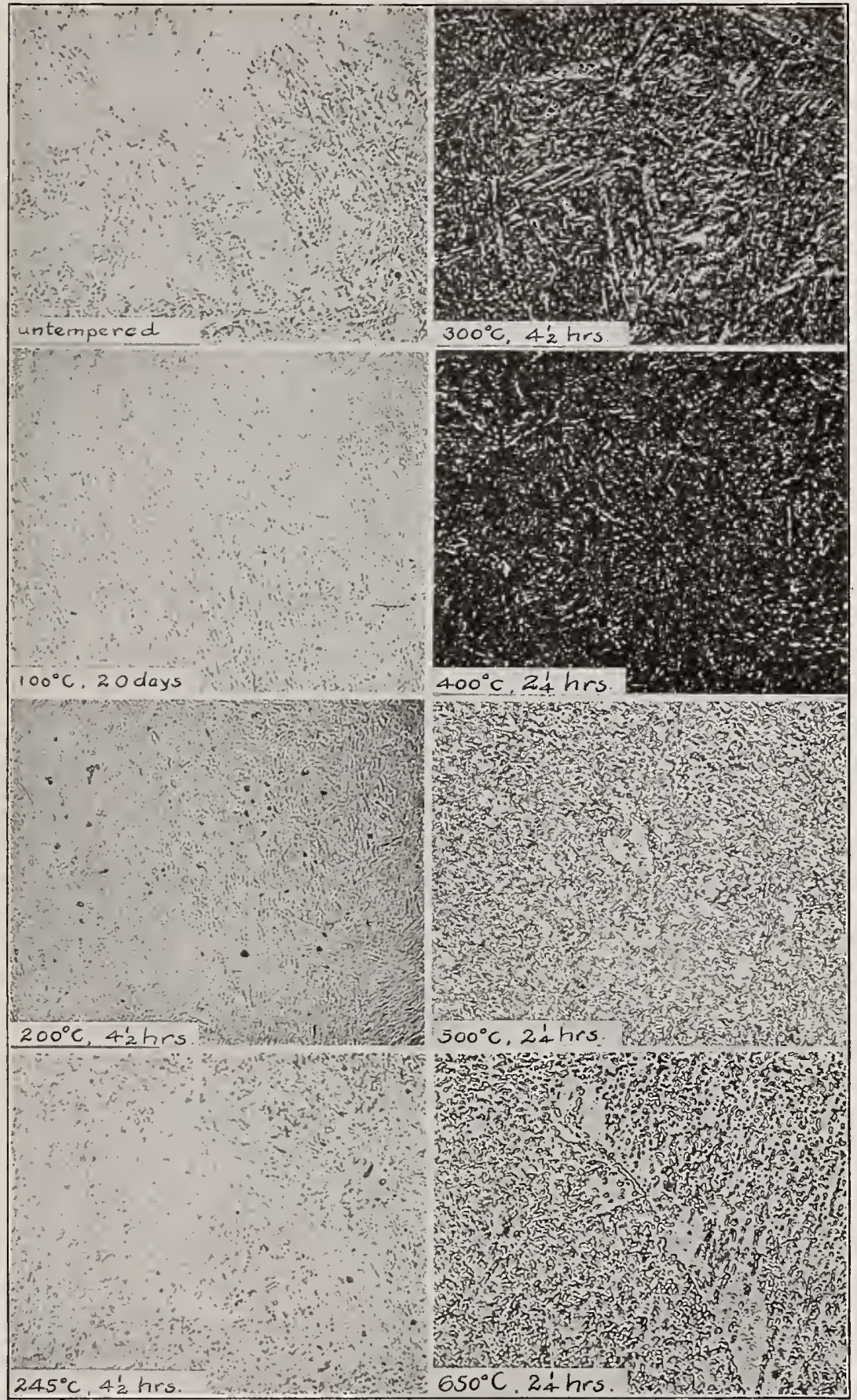

FIG. 7.-Effect of tempering upon the microstructure of 0.70 per cent carbon steel quenched in water from $1050^{\circ} \mathrm{C}$. after 2 hours at this temperature; $\times 500$.

Etching reagent, see p. $37^{8}$.

$111668^{\circ}-22-3$ 
ence of austenite, particularly if the material has been slightly tempered. (Fig. I 5.) Hanemann ${ }^{8}$ has called attention to some of these features in the structure of martensite. However, all of the quenching temperatures used by Hanemann in his investigation of this point were considerably above those of the corresponding solidus, so that the steel must have been heated to a temperature of incipient fusion before quenching. On account of the uncertainty thus introduced, a general conclusion that the phenomena observed were necessarily only those resulting from the change of the austenite into martensite does not appear to be warranted. In the present investigation this uncertainty was eliminated by using a series of quenching temperatures, the highest of which was considerably below that of the solidus. (Fig. 1.) The results obtained serve to substantiate and confirm the conclusions reached by Hanemann as a result of his incomplete observations.

The fact that there is a redistribution of the carbon, which is very different from that originally obtaining throughout the space previously occupied by the uniform austenitic crystal, is shown conclusively by examining the specimen after it has been tempered sufficiently so that the particles of cementite are large enough to respond to the sodium picrate etching test. Figure I 4 shows unquestionably the fact that the "needles" are of a lower carbon content than is the "filling" material. The investigations of Chevenard, ${ }^{9}$ by means of the dilatometer, have shown that the formation of martensite during the quenching of a specimen of steel is accomplished at a relatively low temperature, I 50 to $300^{\circ} \mathrm{C}$. A redistribution of the carbon throughout the individual crystals of a steel at as low a temperature as this appears almost inconceivable; however, no other explanation is available. It is well known that in the austenitic state the carbon is uniformly distributed within the individual grains; each grain is uniform in composition throughout and does not differ in composition from the neighboring ones. The theory that there are two allotropic forms of martensite, the $\alpha$ and $\beta$, which differ in their etching properties, their response to tempering, and their relative dilatation has been advanced by Honda ${ }^{10}$ in his explanation of observed differences in the expansion of steels hardened under different conditions. It was suggested by Le Chatelier in the discussion

\footnotetext{
8 See note 3.

${ }^{9}$ Pierre Chevenard, Mrécanisme de la trempe des aciers des carbone, Rév. de Met. Mémoires, 16, p. 18 ; rorg.

${ }^{10} \mathrm{~K}$. Honda, On the Nature of the $A_{1}$ Transformation and a Theory of Quenching, Sci. Reports, Tohoki1 Imp. Uuiversity, Sendai, Japan; also Jour. Iron and Steel Inst., 100, p. 4I 7 ; 1919.
} 


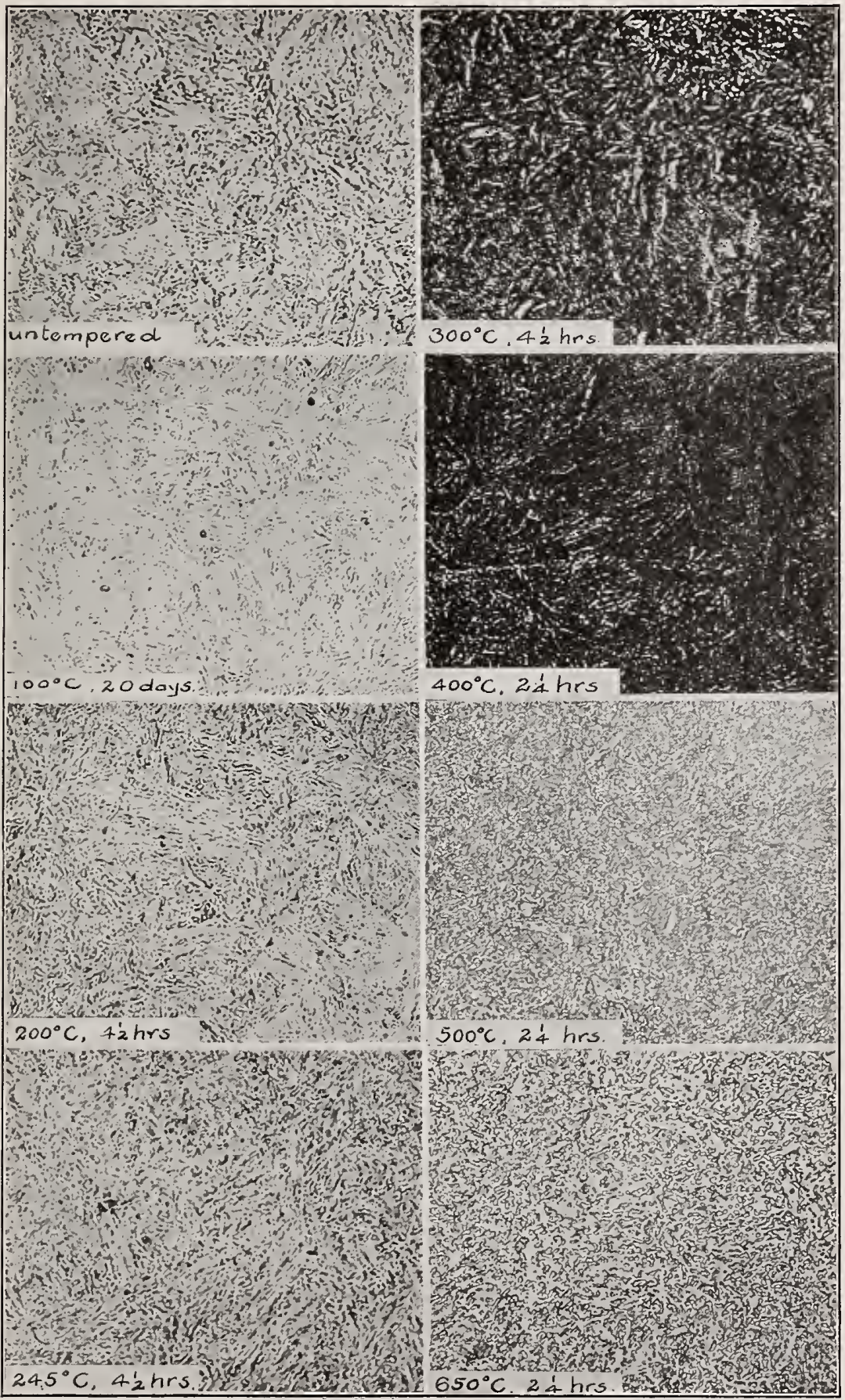

FIG. 8.-Effect of tempering upon the microstructure of 0.85 per cent carbon steel quenched in water from $850^{\circ} \mathrm{C}$, after 15 minutes at this temperature; $\times 500$.

Etching reagent, see p. 378 . 


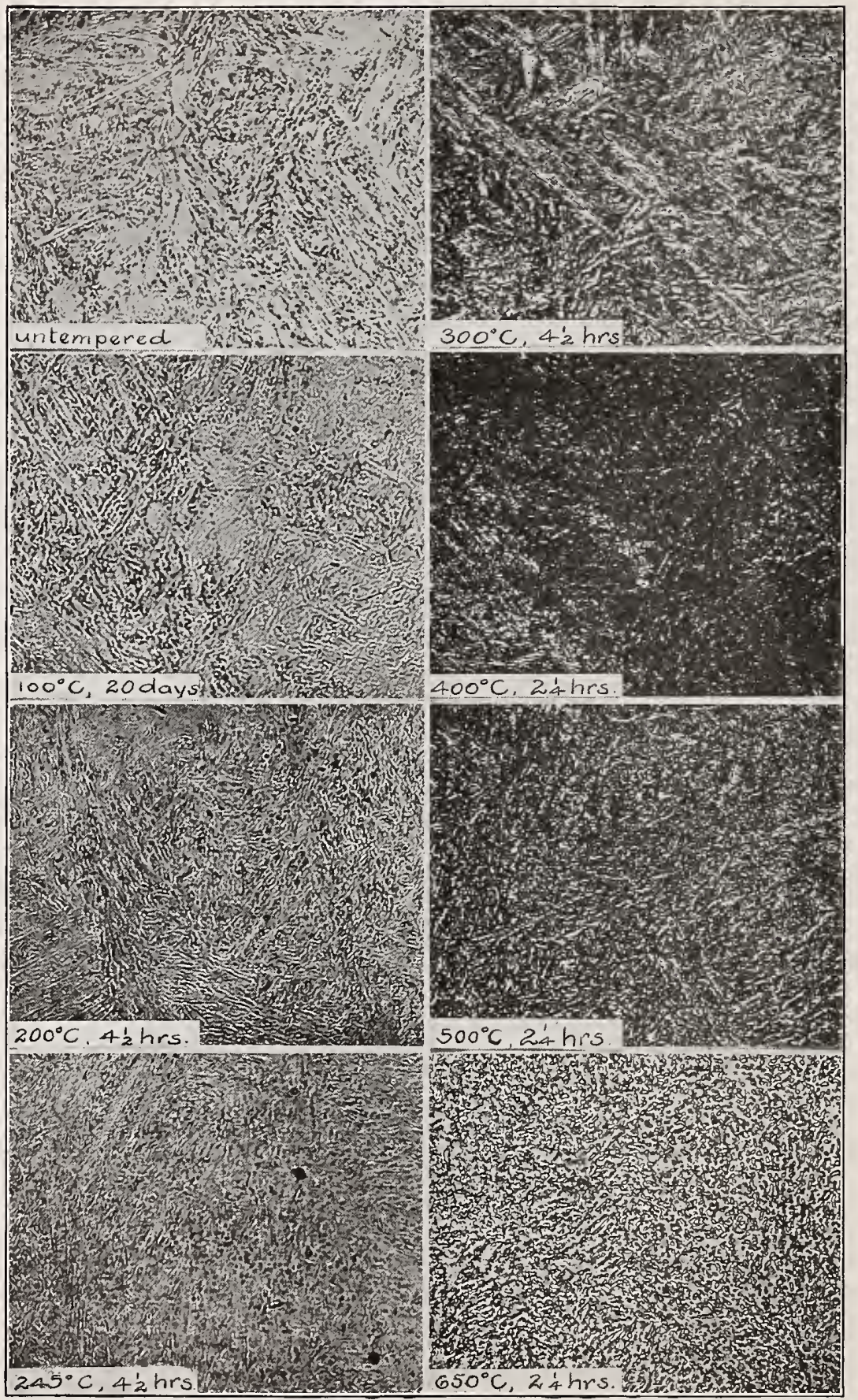

Fig. 9.-Effect of temperature upon the microstructure of 0.85 per cent carbon steel quenched in water from $1050^{\circ} \mathrm{C}$. after 2 hours at this temperature; $\times 500$.

Etching reagent, see p. 378 . 
of this article that the $\alpha$ form of martensite was probably the unchanged austenite which remained in some of the steel after quenching. The variation in chemical composition throughout the martensitic matrix, as discussed above, may also have an important bearing on this matter.

A casual reading of some of the recent articles concerning the atomic structure of martensite and austenite ${ }^{11}$ might lead one to conclude that martensite is relatively as simple in structure as is austenite. However, the term as generally employed is used to refer to the entire structure which results from the austenitic transformation and includes both "needles" and "filling material." The fact that the needles are quite different in chemical composition from the filling material has been discussed above. They also show differences of a physical nature, one of the most striking being revealed by the "quenching" cracks which often form in the larger ones. In addition to the commonly observed quenching cracks, which are intercrystalline, in that they form between what constituted grains when the steel was in the austenitic state, many of the specimens of high carbon content quenched from the high temperatures showed a second type of cracks. In most cases these were short and extended transversely across the needles and terminated abruptly; that is, did not extend into adjacent needles. (Fig. 5.) In many instances several cracks, all approximately parallel to each other, were observed within the same needle. A few extending parallel to the length of a needle were found at times; these, however, were very much fewer in number than the transverse ones. Most of the cracks observed were short and straight. In those cases in which branched or angled ones occurred an explanation was usually at hand in that such cracks were generally found to be located near the junction of two needles, and consequently the crack branched so as to adapt itself to the orientation of each of them. The fact that these cracks bear a rather definite and characteristic relation to the dimensions of the needle in which they form suggests that the needle has a rather definite and uniform structure throughout and that it is a unit in itself. It may be noted here also that the number of "intercrystalline" quenching cracks found in the hardened specimens was very much smaller than of the type described above. 


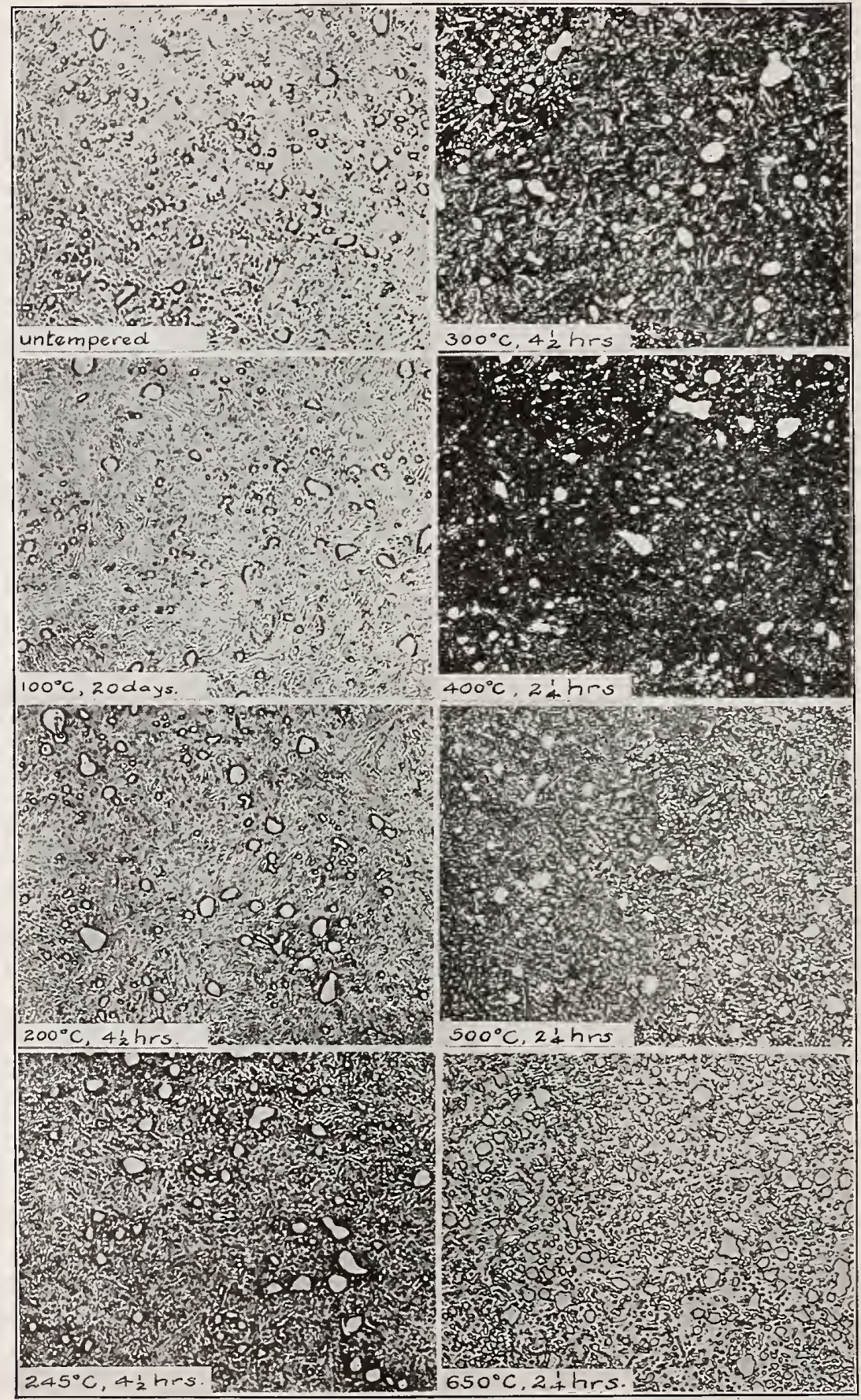

FIG. 10.-Effect of tempering upon the microstructure of $I . I 2$ per cent carbon steel quenched in water from $850^{\circ} \mathrm{C}$. after 4 hours at this temperature.

Etching regeant, see p. 378 . 
(b) Presence of Austenite.-As already explained, the retention of austenite in hardened steels of high carbon content is not of infrequent occurrence. The explanation has been advanced by Honda and his associates ${ }^{12}$ that the formation of cracks in steels during hardening by quenching necessitates the retention of a certain amount of austenite in the martensite, even in steels of relatively low carbon content; for example, 0.28 per cent. No evidence was presented in the published micrographs to substantiate the theory; however, specimens typical of the six compositions used in the present investigation were examined for the presence of austenite after vigorous quenching, by the methods to be described later. Only in the steel of highest carbon content (I.I2 per cent) was this constituent found in any appreciable amount. In the steel of eutectoid composition only very slight traces of austenite were detected and none at all in the other steels. In order to reconcile these results with the explanation offered by Honda, it would be necessary to assume that the austenite, if present, exists in an ultra-microscopic form and hence could not be detected.

Whether the retention of austenite results entirely from the enrichment in carbon of certain portions of the mother crystal by the redistribution of carbon during the selective process of martensite formation, or whether the stresses resulting from the expansion accompanying this process aid in its retention, is a question of interest and of considerable importance. The investigation, however, offers no new evidence on this point. It was noted, however, that in the specimens of a very coarse martensitic structure, produced by quenching from a high temperature, the amount of austenite retained was very considerably more than that in the same steels quenched from a lower temperature. Likewise in such specimens greater variations in the distribution of the carbon exist than in the finer martensite.

The effect of tempering upon the retained austenite is best considered in the discussion of the tempering changes occurring in martensite.

(c) Changes in Structure Upon Heating.-The changes which take place in martensitic steels upon heating may be very conveniently considered under two headings; those occurring below approximately $250^{\circ} \mathrm{C}$. and those above this temperature. That this distinction is one of real significance and not merely an 


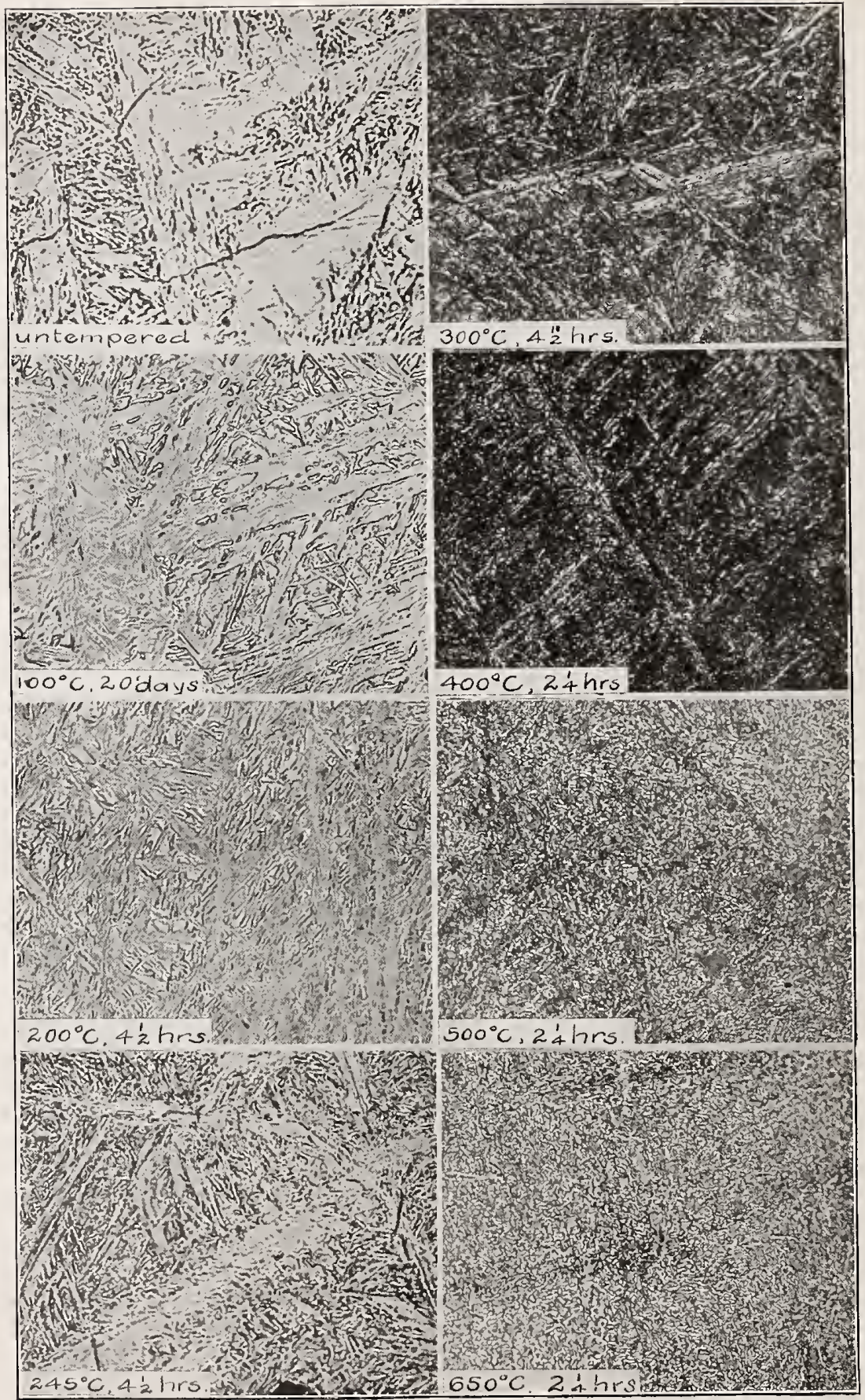

FIG. II.-Effect of tempering upon the microstructure of I.I2 percent carbon steel quenched in water from $I I 50^{\circ} \mathrm{C}$. after $I$ hour at this temperature; $\times 500$.

1 Etching teagent, see p. 378 . 
arbitrary one will become apparent from the discussion which follows.

LoW-TEMPERATURE CHANGES.-The changes resulting from heating martensite at low temperatures, although pronounced, do not produce correspondingly marked visible changes in the microstructure. In general, the difference in the rate of etching of the tempered specimens and the character of the etched surface, as compared to the untempered sample, are the most evident changes noted in the examination of the microstructure. The high carbon steel (I.I 2 per cent C.) will be referred to frequently by way of illustration since, as might be expected, the changes were most pronounced in this one of the six compositions used. Figures 6 to I 2 show the progressive increase in the rate of etching produced by tempering the $0.70,0.85$, and I.I 2 per cent carbon steels when the usual alcoholic picric-acid solution was used, while the opposite effect upon sodium-picrate etching is shown in Figure I4.

The coloring of certain constituents in the microstructure of steel by sodium picrate is essentially a process of oxidation. An investigation now in progress at the Bureau of Standards indicates that similar results may be produced by other oxidizing reagents. The results summarized in Figure I 4 indicate plainly that as martensite approaches a state of structural stability, upon tempering at low temperatures, its tendency toward oxidation decreased and that the coloring of martensite is not simply the result of the surface oxidation of cementite disseminated throughout the material as appears to be the case in fully tempered and annealed steels. It will be noted from Figure I 4 that those portions of the martensitic structure which are of the lower carbon content, as judged by the results obtained in the fully tempered state, are the ones which responded most vigorously to the oxidizing reagent when in the hardened condition. It will also be noted that the austenite retained in the hardened steel did not respond to this etching reagent, a fact which appears to be of considerable significance in any speculation concerning the condition in which the carbon exists in the two constituents.

The readiness of martensitic steel to respond to sodium picrate etching is influenced considerably by the carbon content of the material. In the 0.85 per cent carbon steel the prominent needles of martensite were found to etch less readily than in the specimen just described, so that a very faint etch pattern resulted. The steels of still lower carbon content failed almost entirely to 


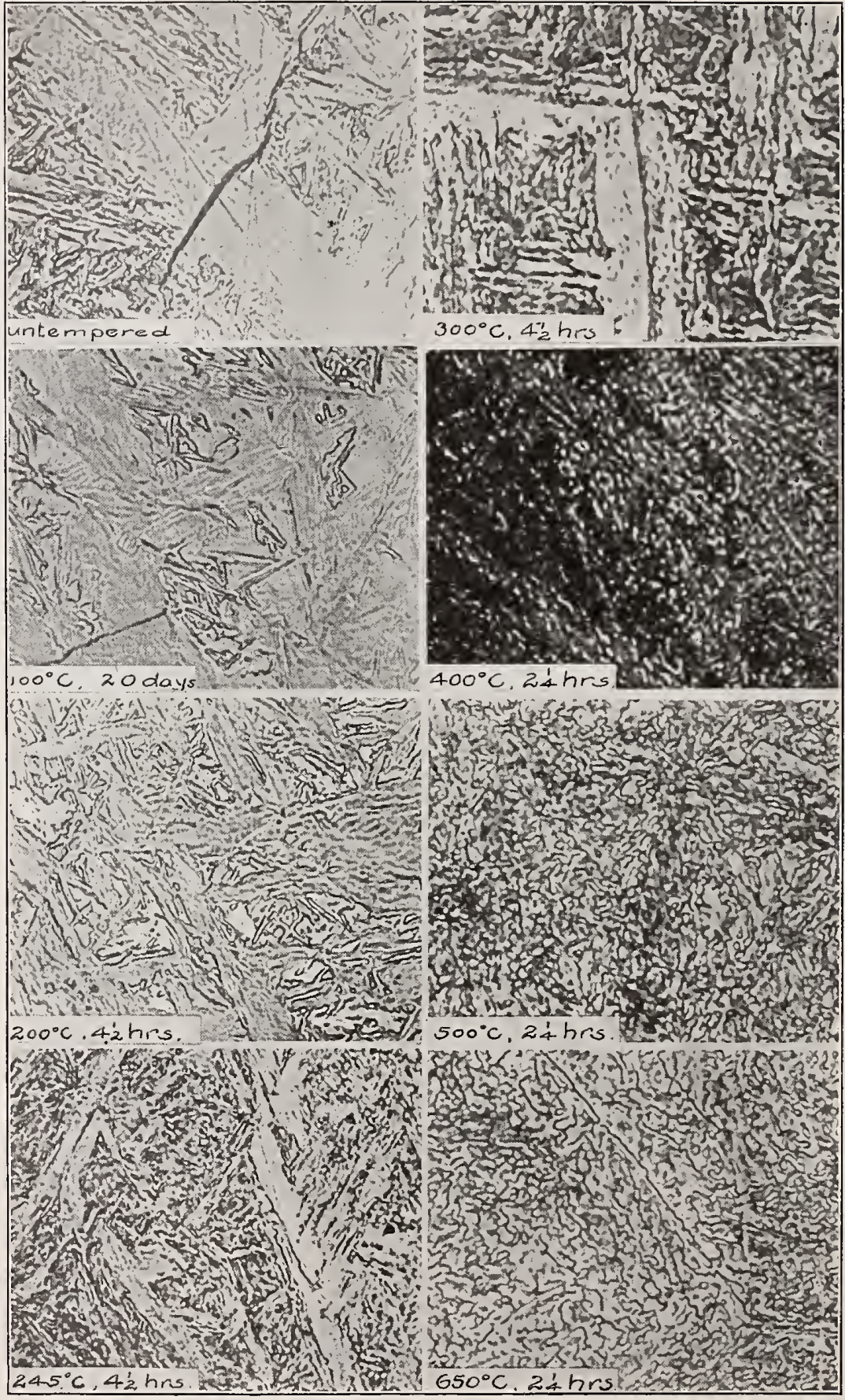

FIG. I2.-Effect of tempering upon the microstructure of I.I2 per cent carbon steel quenched in water from II $50^{\circ} \mathrm{C}$. after I hour at this temperature; $\times 1000$.

Ettching reagent, see p. $37 \mathrm{~s}$. 
respond to the sodium picrate etching when in the hardened state, although when fully tempered, for example, at $650^{\circ} \mathrm{C}$. the cementite readily showed its characteristic coloring in all of them.

With an acid etching reagent the results for the hardened and the slightly tempered specimens were, in general, the reverse of those described above. The prominent "needles" were the most resistant and the "filling material" was more readily etched. (Figs. I2 and 13.) The rate of etching increased progressively as the tempering temperature was increased, and the martensitic structure was found to be revealed most clearly in those specimens which had been slightly tempered, rather than in the initial or fully hardened state. The presence of traces of austenite in a high-carbon steel may be missed entirely if the specimen is examined only in the hardened condition; slight tempering, Ioo or $200^{\circ}$ C., however, changes the relative etching properties of austenite and martensite so that the boundary line between the two readily becomes apparent. The fact that martensite assumes a darker tint as it is tempered slightly, while austenite remains apparently unchanged, aids in identification of the two. This is well shown in Figure I2. The magnification of 1000 diameters employed here is also sufficient to show the slight roughening which the martensite assumes upon etching after being tempered at $200^{\circ} \mathrm{C}$., while slight traces of this may also be seen in the specimen tempered at $100^{\circ} \mathrm{C}$. These low-temperature changes are accompanied by important dimensional changes, usually contraction of the specimen, particularly in high-carbon steel. The behavior of hardened steel in this regard is well known and has received considerable attention.

Figure I I shows clearly the fact that the austenite retained in the hardened steel (I.I 2 per cent C.) persisted after tempering at $200^{\circ} \mathrm{C}$., but that heating at $245^{\circ} \mathrm{C}$. caused disappearance of this constituent. The examination of specimens tempered to different extents, which were subsequently cooled in liquid air, is of interest in showing the persistence of austenite throughout the range of the low-temperature treatment. When a polished specimen of the freshly hardened steel of 1.12 per cent carbon was immersed in liquid air, the surface was very considerably roughened, both martensite and austenite being affected. (Fig. I5.) The same specimen, after being ground and repolished, showed only a very slight roughening upon a second immersion, thus indicating that the change was a permanent one. Subsequent microscopic 


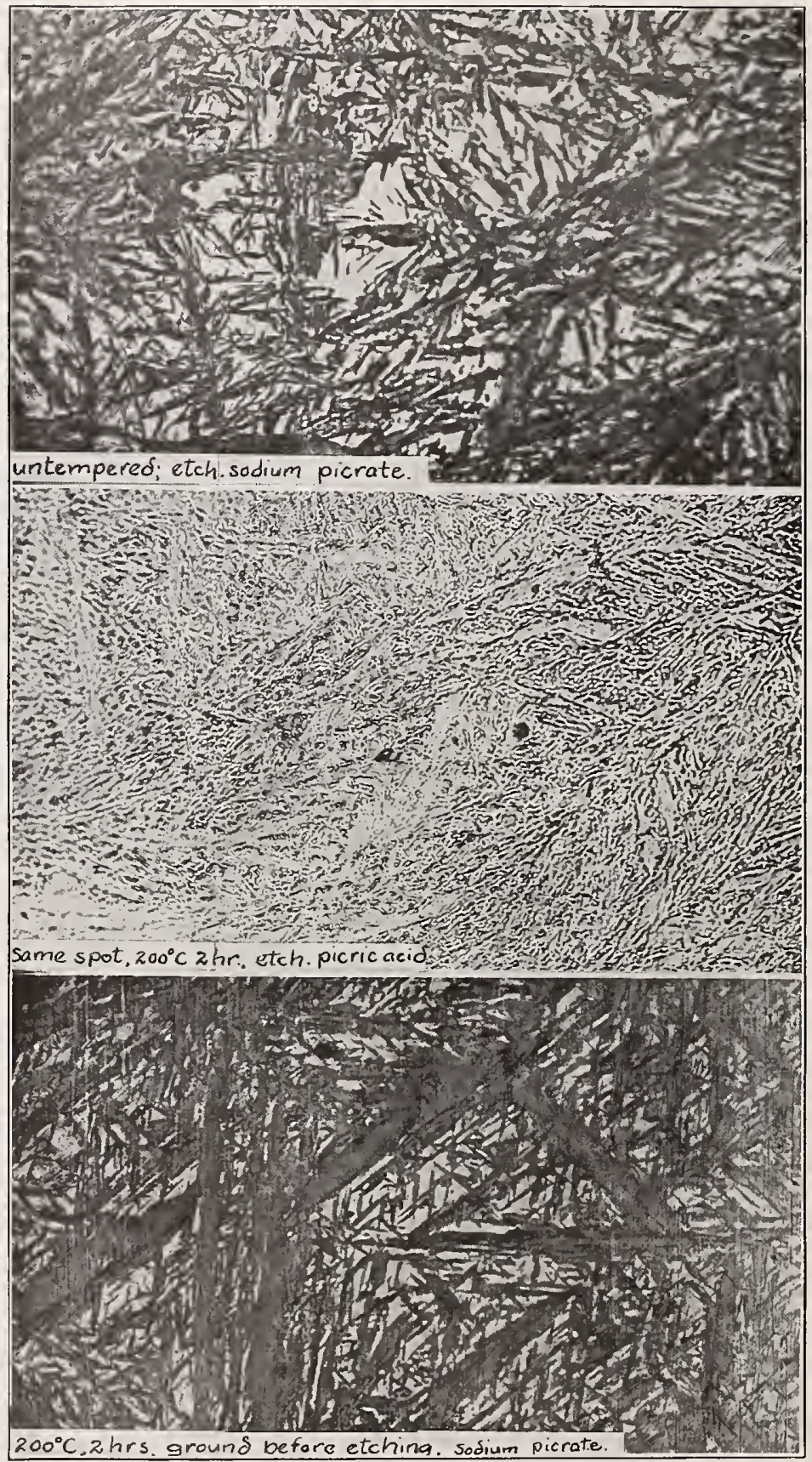

FIG. I3.-Microstructure of $I . I 2$ per cent carbon steel quenched in water from $I 250^{\circ} \mathrm{C}$. after 2 hours at that temperature, illustrating the characteristic behavior toward the two types of etching reagents used; $\times 500$. 


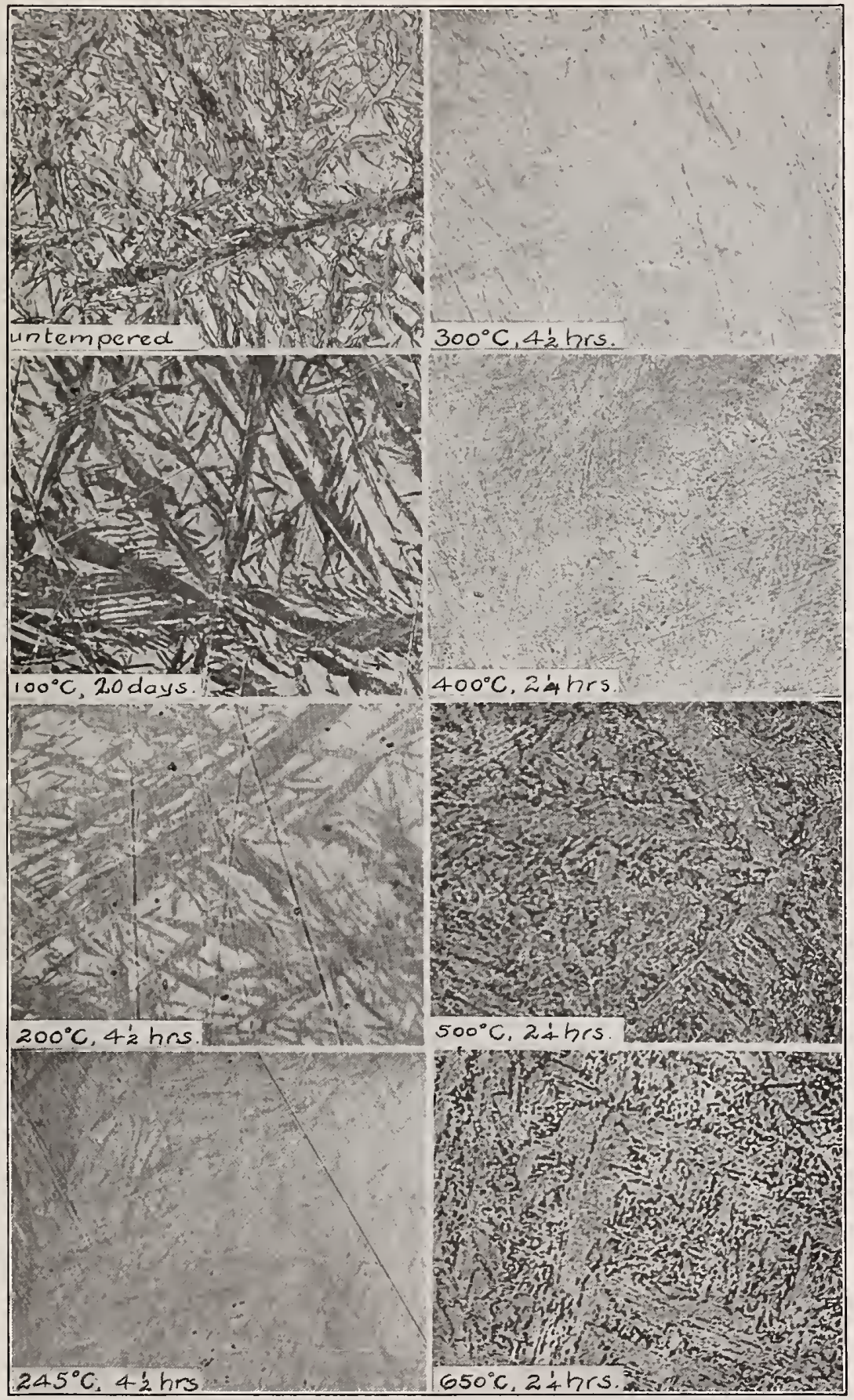

FIG. I4.-Effect of tempering upon the microstructure of 1.12 per cent carbon steel quenched in water from $I I 50^{\circ} \mathrm{C}$. after $I$ hour at this temperature; $\times 500$.

Etching reagent, hot alkaline solution of sodium picrate. 
examination did not reveal any visible changes in the structure of the austenite patches, however, probably on account of the small areas available for observation. When a polished specimen, previously tempered at $100^{\circ} \mathrm{C}$. for 20 days, was immersed in

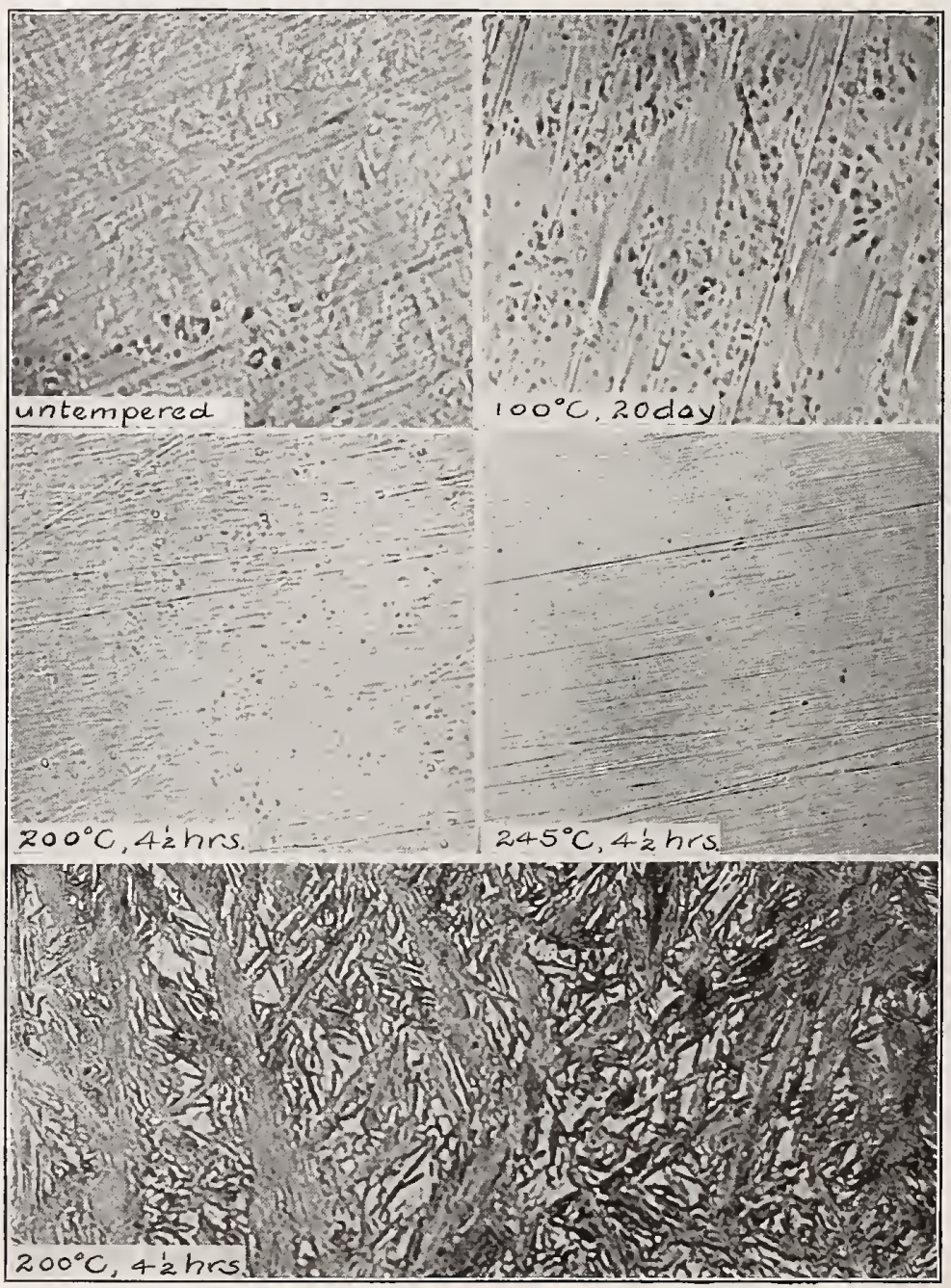

FiG. I 5.-Effect of cooling in liquid air, upon 1.12 per cent carbon steel quenched from $1050^{\circ} \mathrm{C}$. after 2 hours at this temperature and tempered as shown; $\times 500$.

Unetched, except 'e' for which a hot alkaline solution of sodium picrate was used. Note the gradual disappearance of the austenite as the tempering progressed.

liquid air, only the austenite areas responded to the temperature change. A specimen tempered at $200^{\circ} \mathrm{C}$, and cooled in the same way, still showed evidence of the presence of austenite by the roughening of the surface that occurred, whereas specimens 
tempered at $245^{\circ} \mathrm{C}$. showed no evidence whatever of surface roughening upon immersion, thus indicating the completion of the transformation of the residual austenite at some temperature between 200 and $2+5^{\circ} \mathrm{C}$.

A point worthy of mention is illustrated in Figure I5. It will be noted that the relative etching characteristics of martensite and austenite with respect to sodium picrate remained unaffected after cooling the specimen in liquid air. The two constituents could be distinguished readily, even after a tempering period of four and one-half hours at $200^{\circ} \mathrm{C}$., followed by immersion in liquid air, when etched with sodium picrate.

It is quite evident, then, from what precedes, that changes which occur in a hardened steel upon tempering are dependent upon the initial structural condition. Steels containing a considerable amount of austenite will have different properties after tempering from those which are largely martensitic at the start, at least until the temperature of $250^{\circ} \mathrm{C}$. (approximate) has been exceeded. Likewise, the presence of troostite in hardened steels will influence the results upon tempering, although, of course, not in the same manner that austenite does.

TEMPERING AT HIgher TEMPERATURES.-The structural changes which occur in steels when tempered above $250^{\circ} \mathrm{C}$. are much more pronounced than those below this temperature. In general, the final result in all the compositions used was the formation of granules of cementite of such a size as to be seen plainly under moderate magnification. In all of the steels, the cementite granules were plainly visible after tempering at $650^{\circ}$ and in many cases some evidence of the presence of discrete particles was obtained in the specimens tempered at $400^{\circ} \mathrm{C}$. (Fig. I2). However, upon etching with sodium picrate, $500^{\circ} \mathrm{C}$. appeared to be the lowest tempering temperature which rendered the particles large enough to be identified with certainty. Figure I 4 shows this and also the redistribution of carbon which occurred during martensite formation. The coalescence of the cementite into larger particles during the tempering operation - that is, below the $\mathrm{Ac}_{1}$ temperature-does not appear to affect, to any appreciable extent, the distribution of carbon throughout any given section.

The investigation reported by Scott ${ }^{13}$ demonstrated a thermal change in martensitic steels in the neighborhood of $250^{\circ} \mathrm{C}$. This should not be confused with the transformation $\mathrm{A}_{0}$, at $2 \mathrm{I} 5^{\circ} \mathrm{C}$., 

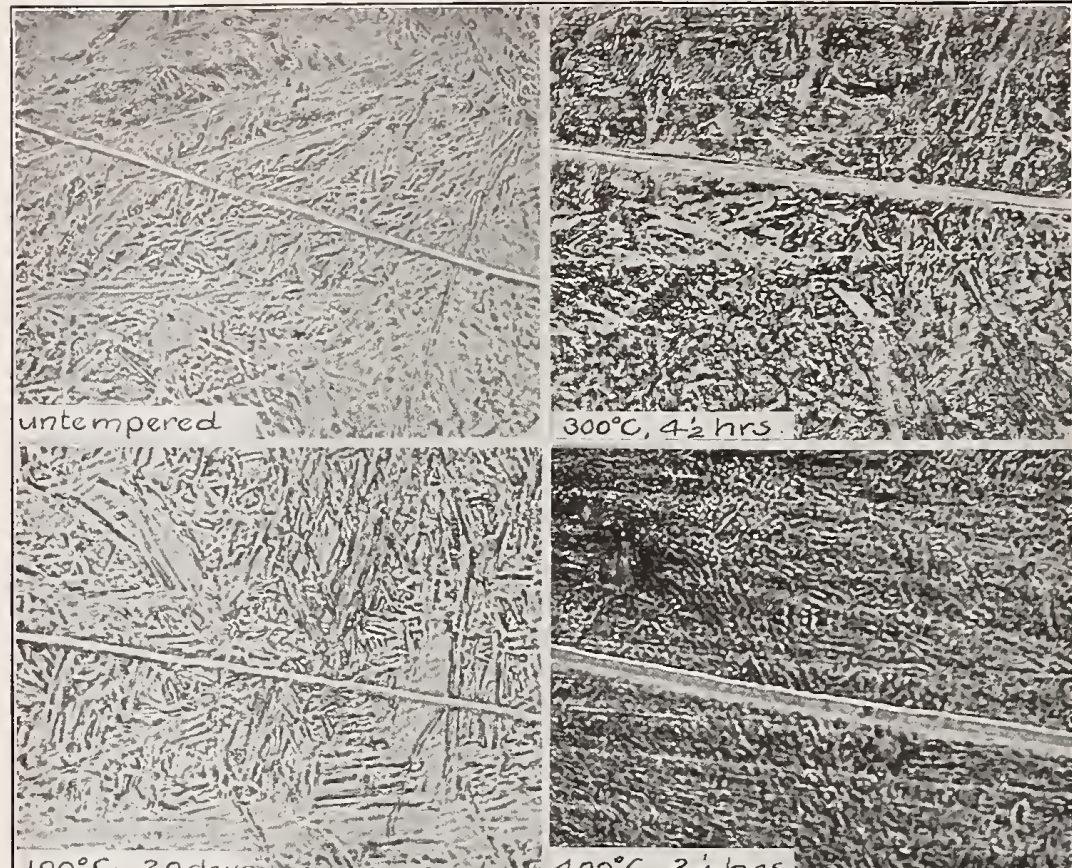

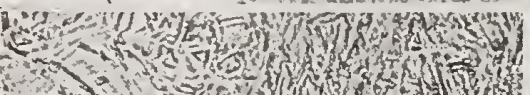
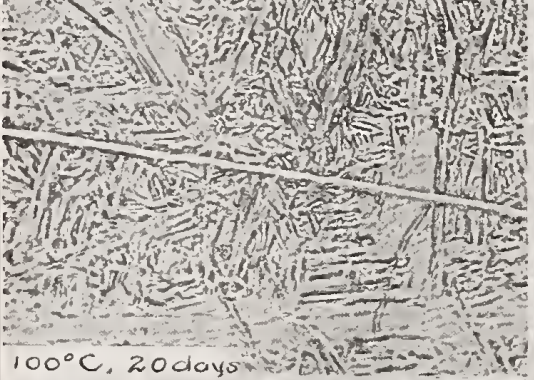

$400^{\circ} \mathrm{C}$ 2 \& hrs thing
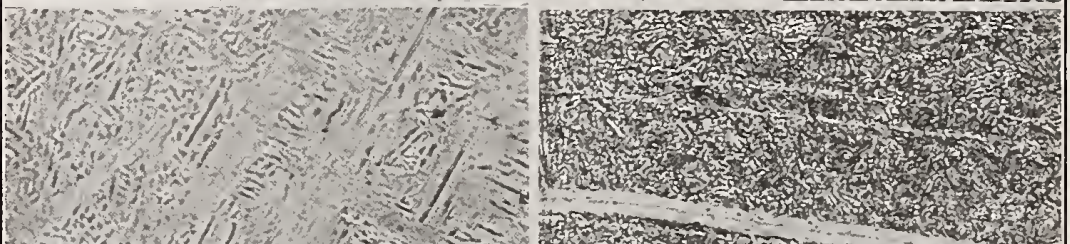

T.t. I. T. ton

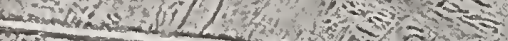

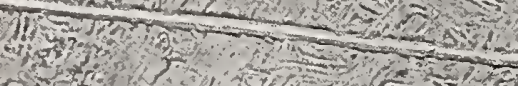

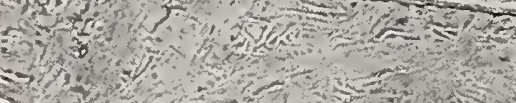
$200^{\circ} \mathrm{C}, 412 \mathrm{hrs}$.

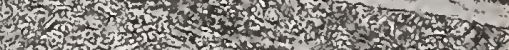
3. 13.

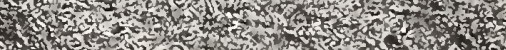
7. $500^{\circ} \mathrm{C}, 24 \mathrm{hrs}$.

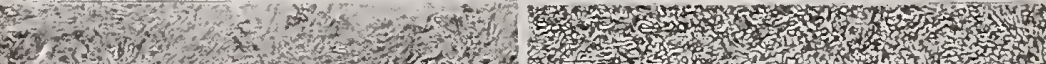
x.

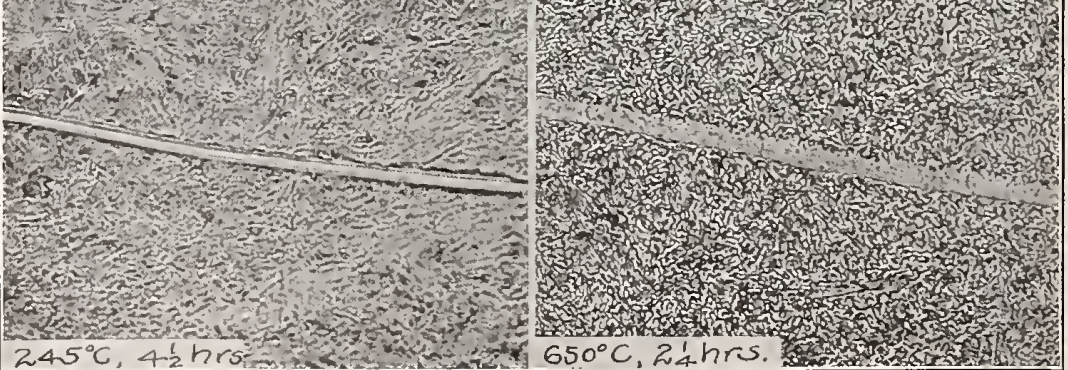

Fig. I6.-Results of the scratch-hardness test upon I.I2 per cent carbon steel quenched in water from $I I 50^{\circ} \mathrm{C}$. after I hour at that temperature and then tempered as indicated; $\times 500$.

Etched with 5 per cent alcoholic picric acid solution before the scratch was drawn. 
reported by Honda and Murakami, ${ }^{14}$ which is a change occurring in the free cementite of annealed steel, or cast iron, and does not necessitate a previous hardening of the steel as does the heat evolution at the slightly higher temperature. It would appear evident, then, that the microstructural changes which occur in hardened steels above the temperature of approximately $250^{\circ} \mathrm{C}$. are quite different in nature from those below.

The pronounced increase in the rate of acid etching of the specimens tempered at 300 and $400^{\circ} \mathrm{C}$. is indicative that the change in structure upon tempering is not a simple progressive one extending throughout the entire temperature range of tempering. Above the temperature of approximately $250^{\circ} \mathrm{C}$., the structural change may properly be regarded as a progressive one resulting in the gradual increase in size of the cementite particles. Below this temperature, however, the changes are essentially different in character from those above. The present investigation does not afford enough evidence to warrant any extended discussion concerning the probable nature of the low-temperature changes in martensite. It is apparent, however, that such changes are of a very different magnitude from those occurring at the higher temperatures.

From the micrographs of Figure I 2, taken at a magnification of I ooo diameters, it appears that the coalescing and growth of the carbide particles proceeds most rapidly in the higher-carbon "filling material" between the needles. In fact some of the needles, formerly martensite, show very little evidence of change after tempering at $300^{\circ} \mathrm{C}$.

\section{CHANGES IN HARDNESS.}

In Figure $\mathrm{I} 7$ have been plotted the average values obtained in the scleroscope-hardness determinations of the 0.70 per cent carbon steel, each value plotted being the average of five determinations each on five different specimens quenched simultaneously, as previously described. The figure indicates that there is no obvious or striking relationship between the scleroscope hardness of a quenched steel and the temperature from which such a steel is quenched, also that the period for which it was heated previous to quenching has no marked effect upon the resulting hardness. A similar conclusion was justified by the results obtained for the other steels of higher carbon content. In making such comparisons it is necessary that the lowest temperature be some-

\footnotetext{
${ }^{14} \mathrm{~K}$. Honda and T. Murakami, On the Thermomagnetic Properties of the Carbide Found in Steels, Sci. Rep. Tohoku Imp. Univ., Sendai, Japan, 6, p. 23.
} 
what higher than the $A_{3}$ transformation, as was the case in the results shown in Figure $I 7$. If the temperature of the $A_{3}$ transformation is not exceeded, variations in hardness are naturally to to be expected. In a somewhat similar investigation, Portevin, ${ }^{15}$ obtained results which he decided would not warrant definite conclusions concerning a marked relationship between the scleroscope hardness of quenched steels and the length of time such steels were heated previous to quenching, whereas many of the other mechanical properties of the steel are very noticeably affected by variations in this factor in their treatment.

As might be expected, steels lower in carbon showed some differences in hardness corresponding to the lower quenching temperatures since the specimens quenched from temperatures below the upper transformation range retained considerable ferrite.

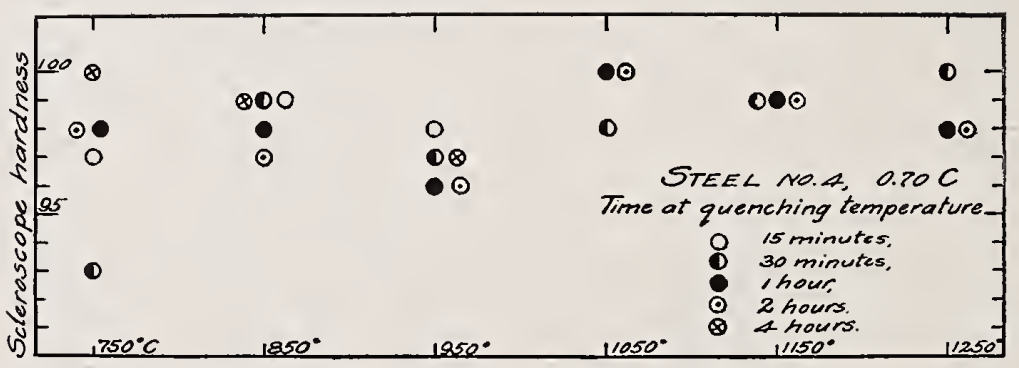

FIG. 17.- Relation between the scleroscope hardness of 0.70 per cent carbon steel and the heating period and quenching temperature.

When quenched from higher temperatures the results for each particular steel were in close agreement and indicated a similar conclusion to that shown in Figure I 7, though of course the hardness numbers obtained varied according to the composition of the steel. From the results obtained the conclusion appeared to be warranted that, provided the steel is fully hardened, the coarseness of the visible "martensitic" structure has no appreciable effect upon the scleroscope hardness of the material. Consequently in the consideration of the effect of tempering upon the hardness, this factor in the hardening treatment could be disregarded.

In Figure I 8 are shown curves summarizing the behavior, with respect to hardness changes, of three of the steels used in the investigation. Each point plotted is the average of 5 determinations, each upon approximately 20 different specimens. The

${ }^{15} \mathrm{~A}$. Portevin, Influence du Temps de Chauffage avant la Trempe sur les Resultats de cette Operation. Rev. de Met. Memoirs, 13, p. 39; 1916. 
steels represented are the two of highest and the one of lowest carbon content. The curves for the other steels closely parallel those shown, the one for steel No. 4 ( 0.70 per cent carbon) lying only very slightly below that of steel No. 5, and the other two somewhat lower in the order of their carbon contents. Howe

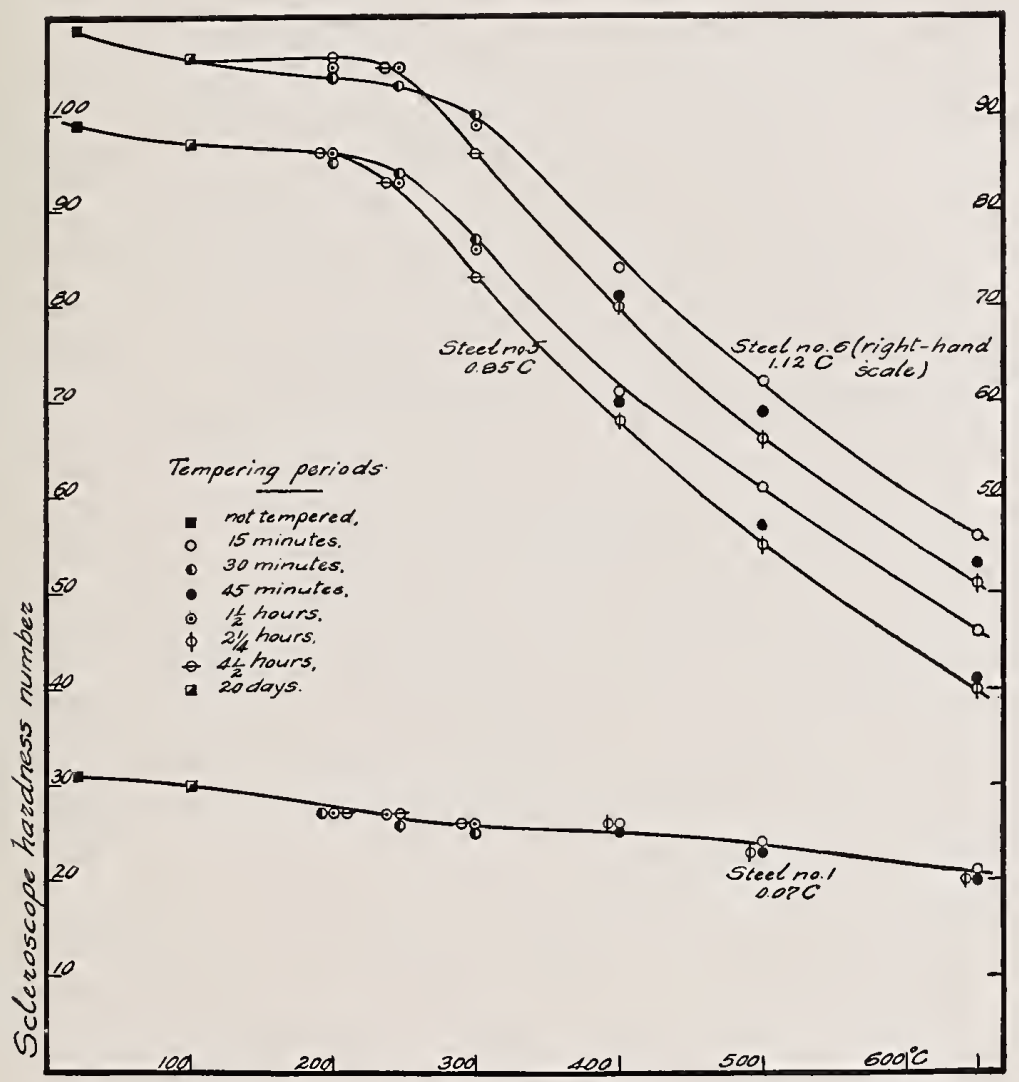

Fig. 18.-Relation between the scleroscope hardness of $0.07,0.85$, and 1.12 per cent carbon steels and the temperature and duration of tempering.

and Levy ${ }^{16}$ have given somewhat similar curves in the report of their investigation of the hardness of eutectoid steel.

The curves for the high-carbon steels indicate very clearly the fact that the changes occurring during tempering at low temperatures are distinctly different from those which ensue at higher temperatures, and it is evident that no very appreciable changes in hardness result until the temperature of $250^{\circ} \mathrm{C}$. (approximate)

${ }^{16}$ H. . M. Howe and A. G. Levy, Notes on the Hardening and Tempering of Eutectoid Carbon Steel and On the Shore Test, A. S. T. M. Proc., 16, p. 5. 
has been reached. The results of the micro-structural examination are thus confirmed. The change in the slope of the curves in the range between 200 and $300^{\circ} \mathrm{C}$. is so marked as to leave no doubt concerning this.

The curve obtained for each of the six steels investigated indicated a gradual though unmistakable decrease in scleroscope hardness upon tempering up to $250^{\circ} \mathrm{C}$. The tempering period has a noticeable effect, however, and in the steel of highest carbon, the specimens heated for four and one-half hours at the low temperatures were harder than those tempered for shorter periods, thus indicating there are two processes, diametrically opposed in effect which tend to take place upon tempering such steels. The gradual softening of the material, as compared to the initial or untempered state, is undoubtedly to be associated with the microchanges in the martensite while the changes which occur in austenite upon heating account for the slight gain in hardness upon prolonged tempering. This gain was not great enough, however, to bring the material back to its initial hardness. The increase in hardness in the 1.12-carbon steel was pronounced enough so that a double curve could be used to represent the effect. Although there was distinct evidence of a similar change in the 0.85 carbon steel, it did not appear to be pronounced enough as to warrant the use of a double curve. In the steels of lower carbon content no evidence of a gain in hardness upon tempering was obtained; likewise there was no indication of austenite found in the microstructure of these steels.

When tempered at the higher temperatures-that is, above $250^{\circ} \mathrm{C}$. - all the steels were found to lose their hardness rapidly and the curves become roughly linear, so that the change in hardness may be considered as approximately proportional to the increase of temperature above the $250^{\circ} \mathrm{C}$. point. The effect of the tempering period is shown by the two branches of the curve, which diverge slightly as the tempering temperature is increased. In general, it may be concluded that, although the precision of the scleroscope is not very high, the results obtained in the study of the hardness changes during tempering strongly confirm the conclusions which the observed changes in the microstructure of the same steels appeared to justify.

The results obtained in the determination of the scratch hardness of steel No. 6 ( I. I 2 per cent carbon) are shown in Figure I6. This method was used principally in the endeavor to show possible 
differences of hardness existing between the various " constituents" which make up the martensitic condition in steel. No significant results were obtained however. The specimens were etched before the scratch was drawn and the irregularities to be noted along the margin of the scratch in some cases are to be considered as the result produced upon the "etching film" on the surface of the specimen rather than of any special significance with respect to the hardness of the material. The average width of the scratch in the different specimens represents very nicely the change in the abrasion-resisting properties of the steel during the various stages of tempering. It will be noted that a considerable difference in the width of the scratch existed in the specimen tempered at $200^{\circ} \mathrm{C}$. as compared with the one at $100^{\circ}$ - a fact which appears to be of some significance in connection with the heat treatment of steel specimens, such as gages, which must show high resistance to abrasion.

\section{SUMMARY.}

I. A study of the microstructure of hardened and tempered carbon steels was carried out on a series of 6 steels varying from 0.07 to I.I2 per cent carbon. These were hardened by water quenching from different temperatures, ranging from 750 to I $250^{\circ} \mathrm{C}$. The effect of heating previous to quenching was also considered. The results produced by tempering were studied in hardened specimens reheated to temperatures varying from 100 to $650^{\circ} \mathrm{C}$, consideration being given to the length of time the specimen was reheated. The investigation consisted largely in microscopic examination, supplemented by a study of the scleroscope hardness of the quenched and of the tempered steels.

2. The coarseness of the martensitic structure appears to be determined almost entirely by the temperature from which the material is quenched; the influence of the period during which the material is heated previous to quenching, at least up to four hours, is relatively very slight as compared with that of temperature.

3. Each crystal of austenite of the hot steel is transformed during the quenching into a system of martensitic plates or "needles," the orientation of the system from each crystal being quite distinct from that produced in the other neighboring crystals. Martensite is formed from austenite in a manner analogous to the freezing of a solid-solution alloy, in that each of the originally 
chemically homogeneous austenite crystals gives rise to a structure that is nonhomogeneous in composition. A redistribution of carbon takes place and the conspicuous martensite plates or "needles" are found to be distinctly lower in carbon than the "filling material." Evidence of this may be obtained from the characteristic behavior of the specimen after complete tempering toward etching reagents. The enrichment in carbon of the filling material between the needles may be great enough so as to allow small patches of austenite to persist in the steel after quenching. Both the 0.85 and I.I2 steels showed austenite, particularly in specimens quenched from the highest temperatures. None of the other steels showed any features, however, which suggested the retention of austenite after quenching.

4. The changes which occur during tempering are considered under two headings - those below and those above $250^{\circ} \mathrm{C}$. The structural changes below this temperature are relatively slight and manifest themselves by the difference in the rate of etching of martensite, particularly in the steels of high carbon content, with respect to alkaline oxidizing reagents.

5. By means of liquid-air treatment it was shown that lowtemperature tempering has only slight effect upon the austenite retained in high-carbon steels after quenching. Between 200 and $250^{\circ} \mathrm{C}$, however, it is transformed entirely and disappears as a constituent of the microstructure in steels tempered to $250^{\circ}$ or above.

6. A relatively pronounced change in structure of hardened steels occurs at approximately $250^{\circ}$. The martensite and austenite, if present, are completely transformed and the steel begins to assume a granular appearance. The rate of etching in acid reagents increases very rapidly soon after this tempering temperature has been reached. Further tempering increases the granular structure, so that after heating at approximately $500^{\circ} \mathrm{C}$. the individual particles of cementite may be detected under the microscope. Fully tempered steels after being etched with a reagent which colors the cementite particles show the unhomogeneity in composition which resulted from the redistribution of carbon which occurred during martensite formation.

7. 'The curves summarizing the scleroscope hardness of steels tempered at various temperatures show a distinct change of slope at approximately $250^{\circ} \mathrm{C}$. Below this temperature the hardness decreases very slightly as compared to the rate of decrease above 
$250^{\circ} \mathrm{C}$. In the steel of highest carbon content used the hardness number after a long tempering period below $250^{\circ} \mathrm{C}$. was distinctly ligher than after a short tempering at the same temperature. The hardening resulting from the gradual change in the austenite neutralizes to some extent the softening in the martensite, which occurs readily upon tempering even at low temperatures. None of the tempered specimens, however, showed a scleroscope hardness number greater than that of the initial hardness directly after quenching, as has been reported by some investigators.

8. An increase in the temperature from which the steels were quenched, provided this was above the $\mathrm{Ac}_{3}$ transformation or the use of a long heating period previous to quenching, produces no marked effect upon the scleroscope hardness of the quenched steel.

9. The evidence afforded by the investigation did not appear to warrant any speculative discussion concerning the ultimate nature of martensite, although several important features were revealed, which must be accounted for in any explanation of the real nature of this constituent.

WASHINGTON, Marcli 20, I922. 



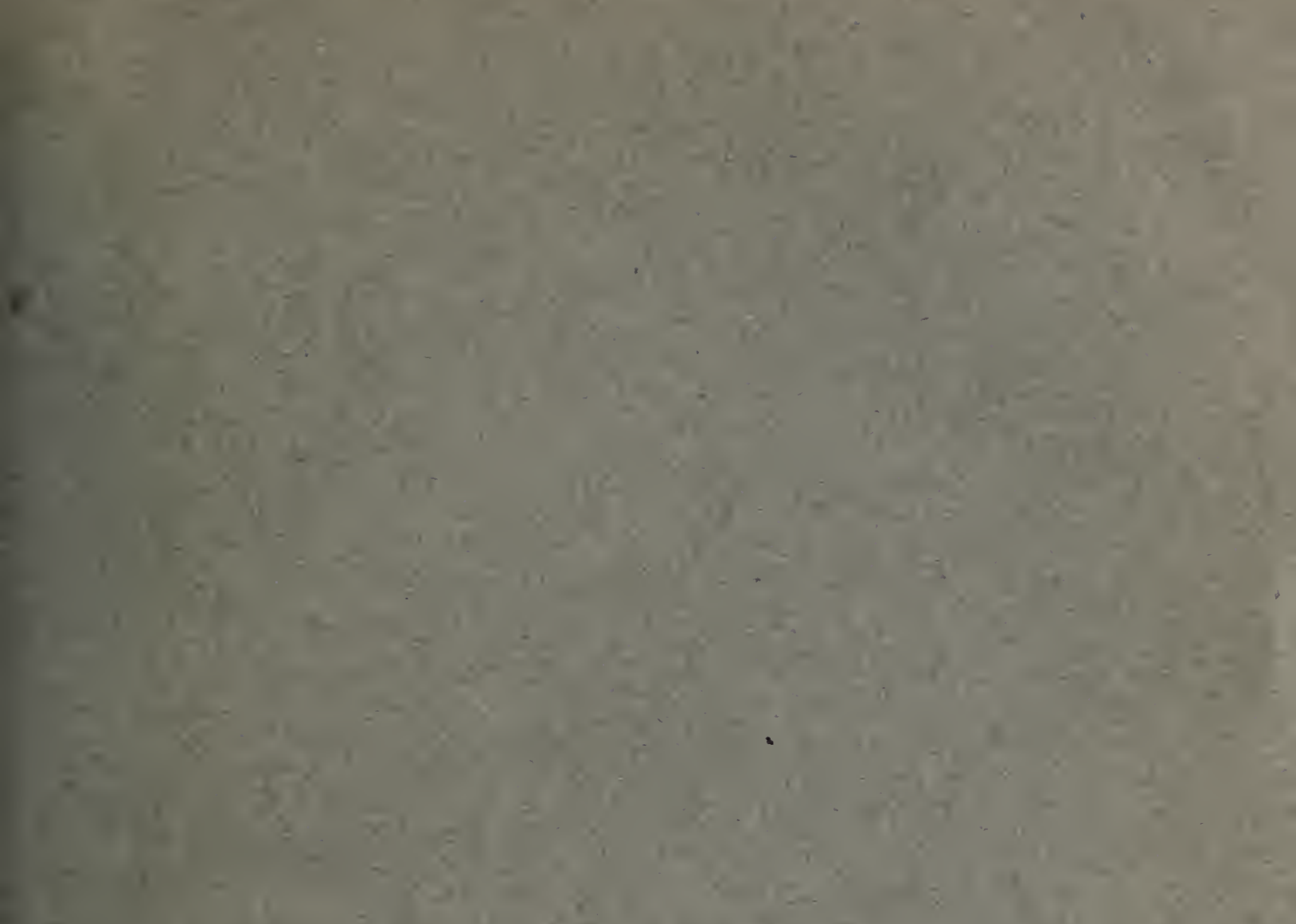

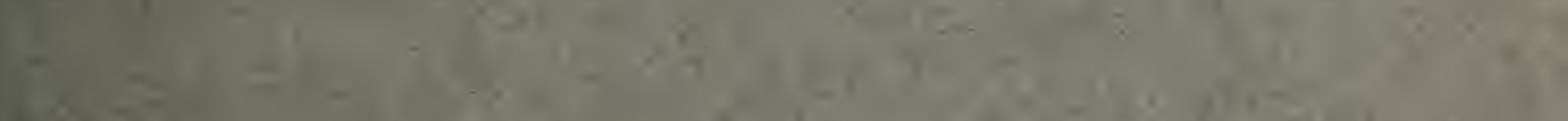

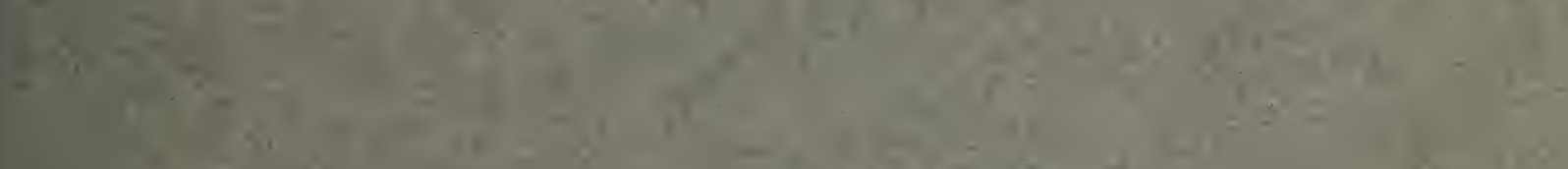

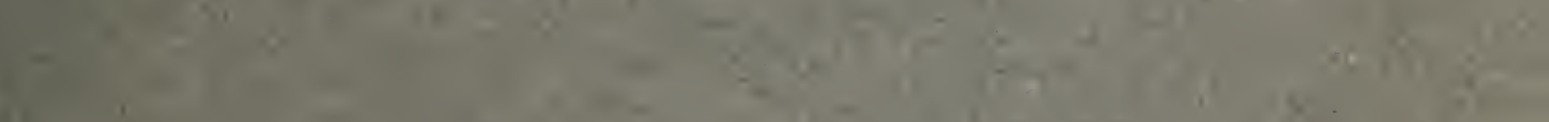

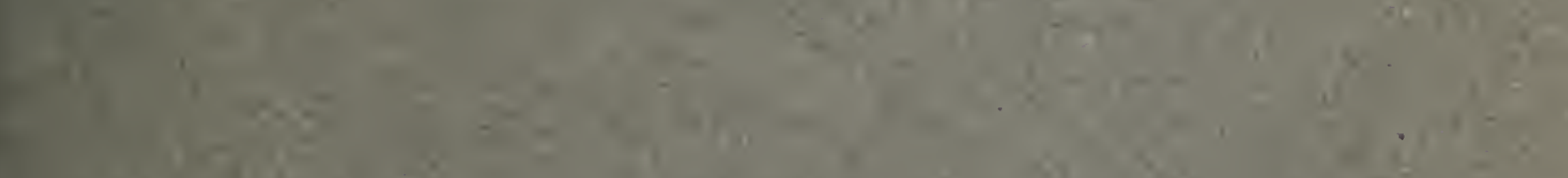

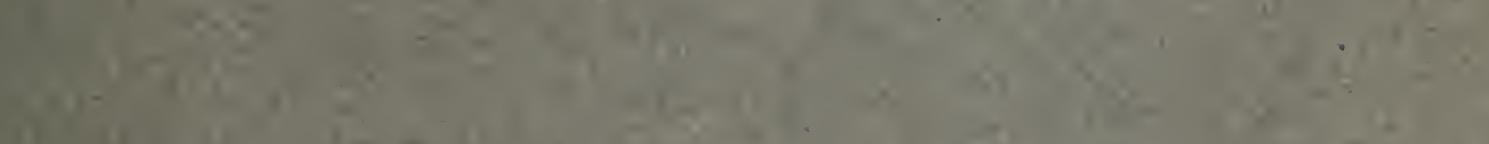

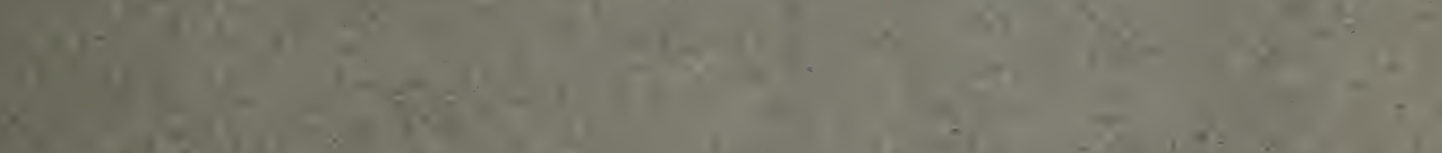
Q

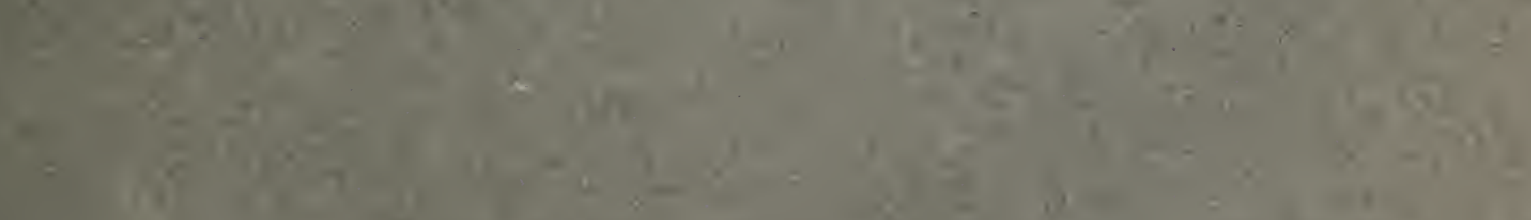

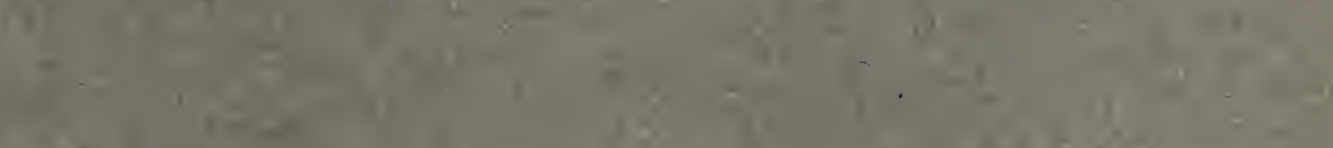

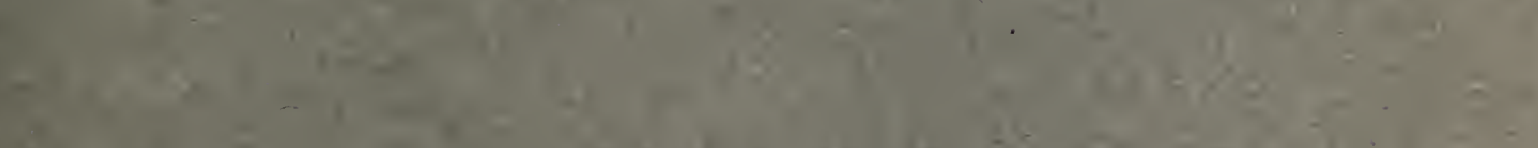

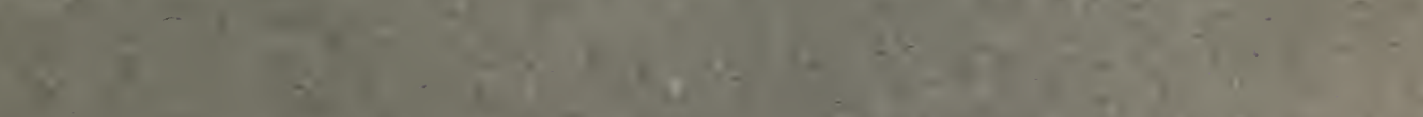

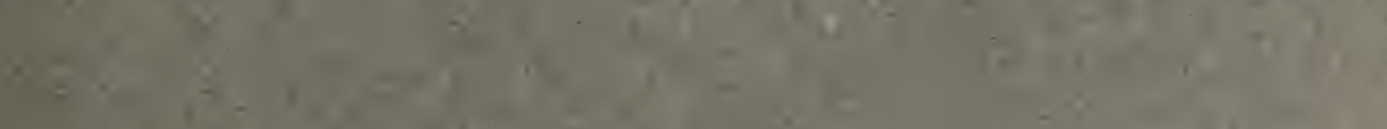

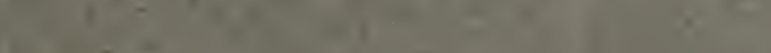

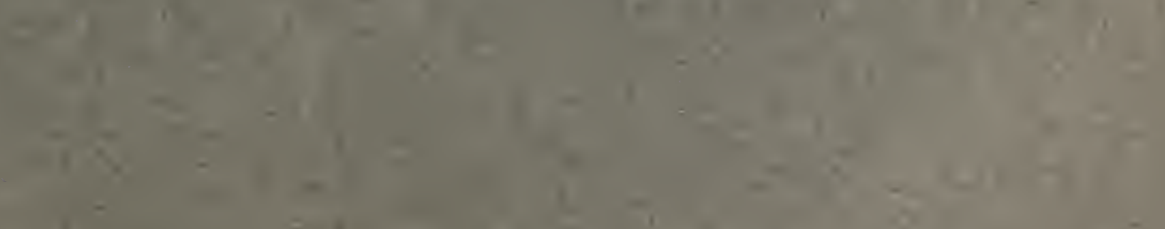

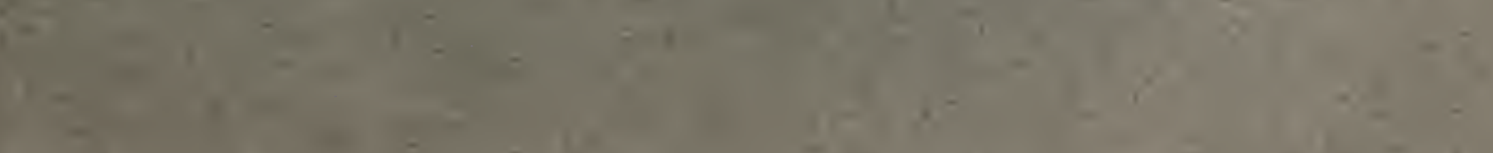


